\title{
Histopathological Image Analysis: A Review
}

\author{
Metin N. Gurcan, Senior Member, IEEE, Laura E. Boucheron, Member, IEEE, Ali Can, \\ Anant Madabhushi, Senior Member, IEEE, Nasir M. Rajpoot, Member, IEEE, and Bulent Yener, Senior Member, IEEE
}

\author{
Methodological Review
}

\begin{abstract}
Over the past decade, dramatic increases in computational power and improvement in image analysis algorithms have allowed the development of powerful computer-assisted analytical approaches to radiological data. With the recent advent of whole slide digital scanners, tissue histopathology slides can now be digitized and stored in digital image form. Consequently, digitized tissue histopathology has now become amenable to the application of computerized image analysis and machine learning techniques. Analogous to the role of computer-assisted diagnosis (CAD) algorithms in medical imaging to complement the opinion of a radiologist, CAD algorithms have begun to be developed for disease detection, diagnosis, and prognosis prediction to complement the opinion of the pathologist. In this paper, we review the recent state of the art CAD technology for digitized histopathology. This paper also briefly describes the development and application of novel image analysis technology for a few specific histopathology related problems being pursued in the United States and Europe.
\end{abstract}

Index Terms-Computer-aided diagnosis, computer-assisted interpretation, digital pathology, histopathology, image analysis, microscopy analysis.

\section{INTRODUCTION AND MOTIVATION}

$\mathbf{T}$ HE widespread use of computer-assisted diagnosis (CAD) can be traced back to the emergence of digital mammography in the early 1990s [1]. Recently, CAD has become a part of routine clinical detection of breast cancer on mammograms at many screening sites and hospitals [2] in

Manuscript received July 20, 2009; revised October 07, 2009. First published October 30, 2009; current version published December 09, 2009. This work was supported in part by the National Cancer Institute under Grants R01 CA134451, R01CA136535-01, ARRA-NCl-3 21CA127186-02S1, R21CA127186-01, R03CA128081-01, and R03CA143991-01, National Library of Medicine R01 LM010119, American Cancer Society, The Children's Neuroblastoma Cancer Foundation, Wallace H. Coulter Foundation, New Jersey Commission on Cancer Research, The Cancer Institute of New Jersey, and the Life Science Commercialization Award from Rutgers University, The Ohio State University Center for Clinical and Translational Science, Department of Defense under Grant W81XWH-07-1-0402.

M. N. Gurcan is with the Department of Biomedical Informatics, The Ohio State University, Columbus, OH 43210 USA (e-mail: metin.gurcan@osumc. edu).

L. E. Boucheron is with the Klipsch School of Electrical and Computer Engineering, New Mexico State University, Las Cruces, NM 88003 USA (e-mail: lboucher@nmsu.edu).

A. Can is with the Global Research Center, General Electric Corporation, Niskayuna, NY 12309 USA (e-mail: can@research.ge.com).

A. Madabhushi is with the Biomedical Engineering Department, Rutgers University, Piscataway, NJ 08854 USA (e-mail: anantm@ rci.rutgers.edu).

N. M. Rajpoot is with the Department of Computer Science, University of Warwick, Coventry, CV4 7AL, U.K. (e-mail: N.M.Rajpoot@warwick.ac.uk)

B. Yener is with the Computer Science Department, Rensselaer Polytechnic Institute, Troy, NY 12180 USA (e-mail: yener@cs.rpi.edu)

Digital Object Identifier 10.1109/RBME.2009.2034865 the United States. In fact, CAD has become one of the major research subjects in medical imaging and diagnostic radiology. Given recent advances in high-throughput tissue bank and archiving of digitized histological studies, it is now possible to use histological tissue patterns with computer-aided image analysis to facilitate disease classification. There is also a pressing need for CAD to relieve the workload on pathologists by sieving out obviously benign areas, so that the pathologist can focus on the more difficult-to-diagnose suspicious cases. For example, approximately $80 \%$ of the 1 million prostate biopsies performed in the U.S. every year are benign; this suggests that prostate pathologists are spending $80 \%$ of their time sieving through benign tissue.

Researchers in both the image analysis and pathology fields have recognized the importance of quantitative analysis of pathology images. Since most current pathology diagnosis is based on the subjective (but educated) opinion of pathologists, there is clearly a need for quantitative image-based assessment of digital pathology slides. This quantitative analysis of digital pathology is important not only from a diagnostic perspective, but also in order to understand the underlying reasons for a specific diagnosis being rendered (e.g., specific chromatin texture in the cancerous nuclei which may indicate certain genetic abnormalities). In addition, quantitative characterization of pathology imagery is important not only for clinical applications (e.g., to reduce/eliminate inter- and intra-observer variations in diagnosis) but also for research applications (e.g., to understand the biological mechanisms of the disease process).

A large focus of pathological image analysis has been on the automated analysis of cytology imagery. Since cytology imagery often results from the least invasive biopsies (e.g., the cervical Pap smear), they are some of the most commonly encountered imagery for both disease screening and biopsy purposes. Additionally, the characteristics of cytology imagery, namely the presence of isolated cells and cell clusters in the images and the absence of more complicated structures such as glands make it easier to analyze these specimens compared to histopathology. For example, the segmentation of individual cells or nuclei is a relatively easier process in such imagery since most of the cells are inherently separated from each other.

Histopathology slides, on the other hand, provide a more comprehensive view of disease and its effect on tissues, since the preparation process preserves the underlying tissue architecture. As such, some disease characteristics, e.g., lymphocytic infiltration of cancer, may be deduced only from 
a histopathology image. Additionally, the diagnosis from a histopathology image remains the "gold standard" in diagnosing considerable number of diseases including almost all types of cancer [3]. The additional structure in these images, while providing a wealth of information, also presents a new set of challenges from an automated image analysis perspective. It is expected that the proper leverage of this spatial information will allow for more specific characterizations of the imagery from a diagnostic perspective.

The analysis of histopathology imagery has generally followed directly from techniques used to analyze cytology imagery. In particular, certain characteristics of nuclei are hallmarks of cancerous conditions. Thus, quantitative metrics for cancerous nuclei were developed to appropriately encompass the general observations of the experienced pathologist, and were tested on cytology imagery. These same metrics can also be applied to histopathological imagery, provided histological structures such as cell nuclei, glands, and lymphocytes have been adequately segmented (a complication due to the complex structure of histopathological imagery). The analysis of the spatial structure of histopathology imagery can be traced back to the works of Wiend et al. [4], Bartels [5] and Hamilton [6] but has largely been overlooked perhaps due to the lack of computational resources and the relatively high cost of digital imaging equipment for pathology. However, spatial analysis of histopathology imagery has recently become the backbone of most automated histopathology image analysis techniques. Despite the progress made in this area thus far, this is still a large area of open research due to the variety of imaging methods and disease-specific characteristics.

\section{A. Need for Quantitative Image Analysis for Disease Grading}

Currently, histopathological tissue analysis by a pathologist represents the only definitive method (a) for confirmation of presence or absence of disease and (b) disease grading, or the measurement of disease progression. The need for quantitative image analysis in the context of one specific disease (prostate cancer) is described below. Similar conclusions hold for quantitative analysis of other disease imagery.

Higher Gleason scores are given to prostate cancers, which are more aggressive, and the grading scheme is used to predict cancer prognosis and help guide therapy. The Gleason grading system is based solely on architectural patterns; cytological features are not evaluated. The standard schematic diagram created by Gleason and his group (see Fig. 1) separated architectural features into 1 of 5 histological patterns of decreasing differentiation, pattern 1 being most differentiated and pattern 5 being least differentiated. The second unique feature of Gleason grading is that grade is not based on the highest (least differentiated) pattern within the tumor. Recently several researchers have reported discrepancies with the Gleason grading system for grading prostate cancer histopathology. Many researchers have found grading errors (both under- and over-grading) in prostate cancer studies [7]-[11]. Similar issues with cancer grading have been reported for other diseases such as breast cancer [12].

\section{Gleason Scale}

Well differentiated

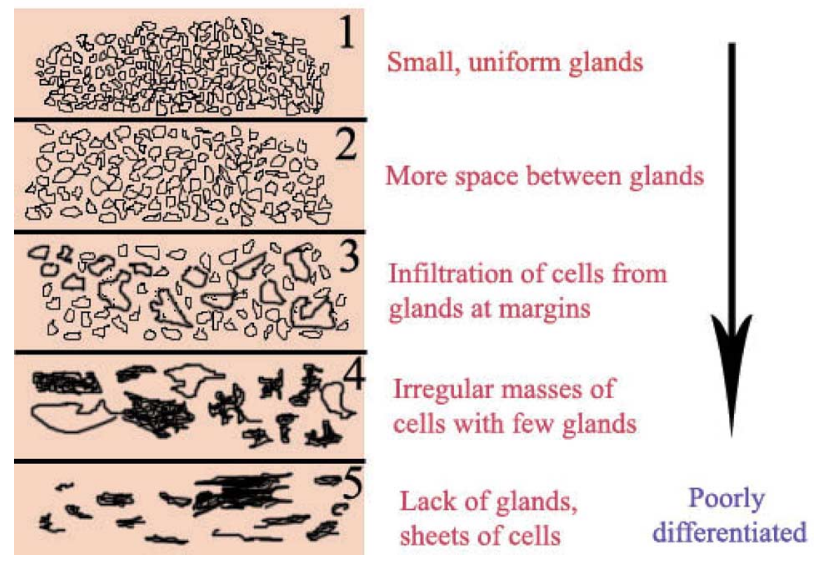

Fig. 1. Schema showing different cancer grades prevalent in prostate cancer.

In light of the above, Luthringer et al. [13] have discussed the need for changes to be made to Gleason grading system. In late 2005, the International Society of Urologic Pathologists in conjunction with the World Health Organization (WHO) made a series of recommendations for modifications to the Gleason grading system, including reporting any higher grade cancer, no matter how small quantitatively.

Luthringer et al. [13] have also suggested the need for re-evaluation of original biopsy material by a highly experienced pathologist which could help guide patient management. Stamey et al. [14] discussed need for developing methods to accurately measure cancer volume and better estimate prostate cancer to better predict progression of cancer. King et al. [8] has similarly called for developing a methodology to help reduce pathologic interpretation bias which would likely result in significantly improved accuracy of prostate cancer Gleason grading.

\section{B. Differences in CAD Approaches Between Radiology and Histopathology}

While CAD is now being used in radiology in conjunction with a wide range of body regions and a variety of imaging modalities, the preponderant question has been: can CAD enable disease detection? Note that this question, as opposed to more diagnostic questions, is motivated by the inherent limitation in spatial resolution of radiological data. For instance, in mammography, CAD methods have been developed to automatically identify or classify mammographic lesions. In histopathology, on the other hand, simply identifying presence or absence of cancer or even the precise spatial extent of cancer may not hold as much interest as more sophisticated questions such as: what is the grade of cancer? Further, at the histological (microscopic) scale one can begin to distinguish between different histological subtypes of cancer, which is quite impossible (or at the very least difficult) at the coarser radiological scale.

It is fair to say that since CAD in histopathology is still evolving, the questions that researchers have started to ask of pathology data are not as well articulated as some of the problems being investigated in radiology. A possible reason for this 
is that image analysis scientists are still trying to come to terms with the enormous density of data that histopathology holds compared to radiology. For instance, the largest radiological datasets obtained on a routine basis are high-resolution chest CT scans comprising approximately $512 \times 512 \times 512$ spatial elements or $\sim 134$ million voxels. A single core of prostate biopsy tissue digitized at $40 \times$ resolution is approximately $15000 \times 15000$ elements or $\sim 225$ million pixels. To put this in context, a single prostate biopsy procedure can comprise anywhere between 12 and 20 biopsy samples or approximately 2.5-4 billion pixels of data generated per patient study. Due to their relatively large size and the content, these images frequently need to be processed in a multiresolution framework.

Also, while radiological CAD systems mostly deal with grayscale images, histological CAD systems often need to process color images. Furthermore, with the recent advent of multispectral and hyperspectral imaging, each pixel in a histopathology section could potentially be associated with several hundred sub-bands and wavelengths.

These fundamental differences in radiology and histopathology data have resulted in specialized CAD schemes for histopathology. While several similar reviews have been published for CAD in medical imaging and diagnostic radiology [15]-[23], to the best of our knowledge no related review has been undertaken for digitized histopathology imagery. A survey for CAD histopathology is particularly relevant given that the approaches and questions being asked of histological data are different from radiological data. The motivation of this paper is to present a comprehensive review of the state-of-the-art CAD methods and the techniques employed for automated image analysis of digitized histopathology imagery.

\section{Organization of This Paper}

We have organized this paper to follow the general image analysis procedures for histopathology imagery. These analysis procedures are generally applicable to all imaging modalities. In Section II, we describe digital pathology imaging modalities including immunofluorescence and spectral imaging and explain the difference between cytopathology and histopathology. In Section III, image preprocessing steps such as color normalization and tissue auto-fluorescence compensation are reviewed. In Section IV, we discuss recent advances in detection and segmentation in histopathological images. Section V is dedicated to feature extraction and selection at different levels, with real-world examples. In Section VI, we review classification and sub-cellular quantification. Finally, in Section VII we discuss some of the potential issues that image analysis of histopathology could be used to address in the future and possible directions for the field in general.

While there are a large number of applicable methods for preprocessing (Section III), detection and segmentation (Section IV), feature extraction and selection (Section V), and classification and sub-cellular quantification (Section VI), we will present here only some common examples. We refer the interested reader to the references contained within the various sections for further reading.

\section{Digital Pathology IMAging Modalities}

\section{A. Histopathology and Cytopathology}

Histopathology is the study of signs of disease using the microscopic examination of a biopsy or surgical specimen that is processed and fixed onto glass slides. To visualize different components of the tissue under a microscope, the sections are dyed with one or more stains. The aim of staining is to reveal cellular components; counter-stains are used to provide contrast. Hematoxylin-Eosin (H\&E) staining has been used by pathologists for over a hundred years. Hematoxylin stains cell nuclei blue, while Eosin stains cytoplasm and connective tissue pink. Due to the long history of H\&E, well-established methods, and a tremendous amount of data and publications, there is a strong belief among many pathologists that $H \& E$ will continue to be the common practice over the next 50 years [24].

Cytology, on the other hand, is related to the study of cells in terms of structure, function and chemistry. Resulting from the least invasive biopsies (e.g., the cervical Pap smear), cytology imagery is the most commonly encountered for both disease screening and biopsy purposes. Additionally, the characteristics of cytology imagery, namely the presence of isolated cells and cell clusters in the images, and the absence of more complicated structures such as glands make it easier to analyze these specimens compared to histopathology.

\section{B. Immuno-Fluorecence Imaging and Multiple Imaging Modalities}

Recently, immuno-fluorescent labeling-based image analysis algorithms have been presented to quantify localization of proteins in tissue [25]-[27]. Commonly used molecular markers are based on chromogenic dyes (such as DAB), or fluorescent dyes (such as Cy dyes or Alexa dyes). Fluorescent dyes have the advantage of multiplexing the dyes to acquire images of multiple proteins. A general overview of molecular labeling, high throughput imaging, and pattern recognition techniques is presented by Price et al. [28].

With current imaging techniques, it is not possible to simultaneously image H\&E dyes and immuno-fluorescent molecular biomarkers due to fluorescent characteristics of the $\mathrm{H} \& \mathrm{E}$ dyes, and due to chemical interactions of H\&E dyes with the fluorescently labeled antibodies. Recently, methods have been developed to facilitate sequential imaging and registration techniques that enable different modalities presented digitally from the same histological tissue section. Additionally, sequential imaging and registration enables imaging of multiple immuno-fluorescent stains acquired in multiple steps rather than conventional simultaneous multiplexing techniques. This allows an order of magnitude increase in the number of molecular markers to be imaged for the same tissue section. These techniques make it possible to explore unexamined relationships between morphology, sub-cellular spatial distribution of proteins, and protein-protein interactions. An example of these techniques is shown in Fig. 2. For brightfield images, hematoxylin stains the nuclei blue [Fig. 2(a)], and for fluorescent images DAPI can be used to stain nuclei [blue channel in 


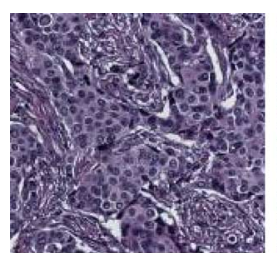

(a)

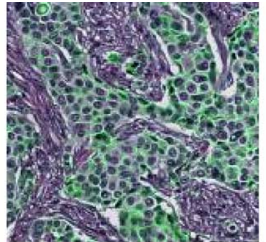

(b)

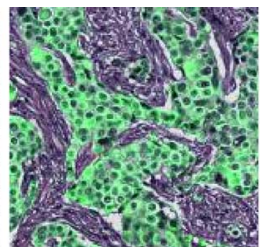

(c)

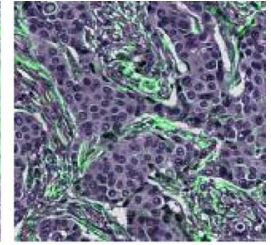

(d)

Fig. 2. (a) H\&E image of a breast tumor tissue. Fluorescently labeled markers superimposed as green color on the H\&E image, (b) $\beta$-catenin, (c) pan-keratin, and (d) smooth muscle $\alpha$-actin, markers.

Fig. 2(a)]. The first nuclei image is set as the reference image and each of the subsequent nuclei images are registered to the reference. Once the transformation parameters are estimated, then all the channels at a sequential step are mapped onto the reference coordinate system. Fig. 2(b)-(d) shows superimposed b-catenin, pan-keratin, and smooth muscle a-actin markers superimposed on the $\mathrm{H} \& \mathrm{E}$ with green pseudo-color [29]. Another recently developed sequential imaging method known as MELC [30] has the ability to produce images of the same specimen with up to 100 proteins by utilizing the photo-bleaching characteristics of the fluorescent dyes.

One of the major problems with such "multichannel" imaging methods is the registration of the multiplexed images, since physical displacements can easily occur during sequential imaging of the same specimen. In [29], the authors used mutual information-based error metrics to register the nuclei images from sequential staining steps. While the fluorescent images include dedicated nuclei channels (such as DAPI), the nuclei images from the H\&E images can be computed using decomposition techniques [31], [32], or using simple ratio or differencing methods that utilize the fact that blue wavelengths are absorbed less than green and red channels by the hematoxylin dye.

\section{Spectroscopic Imaging Modalities for Histopathology}

In recent years, several spectral data acquisition methods have been employed to aid the diagnosis process with additional information about the biochemical makeup of cells and other tissue constituents. Generally, computerized histopathology image analysis takes as its input a three-channel (red, green, and blue or RGB) color image captured by digital imaging equipment (normally a CCD camera) and attempts to emulate the manual analysis and/or provide additional quantitative information to aid in the diagnosis. While analysis of ordinary color images has been shown to be useful, one of the major drawbacks is that only three color channels of the light spectrum are used, potentially limiting the amount of information required for characterizing different kinds of tissue constituents. On the other hand, recently proposed immuno-histochemistry (IHC) methods are not sufficiently well developed for their use in quantitative pathology [33].

Spectral methods offer a relatively inexpensive way of providing a deeper insight into tissue composition. Most of these methods can be categorized into three broad classes: point spectroscopy, spectral imaging, and spectroscopic imaging. Point spectroscopy is a well-established area of study whereby, in the context of histopathology, the chemical composition of a tissue sample is ascertained with the help of the spectrum emitted or absorbed at a specific point on the biopsy. Point spectroscopy methods can employ both visible light and beyond. Spectral imaging, also known as multispectral or hyperspectral imaging, measures intensity of light from the entire optical field after exciting the sample with visible light of varying wavelengths. Spectroscopic imaging combines the strengths of both of the above two methods, building spatial imaging of the human tissue in a multitude of wavelength regimes.

1) Point Spectroscopy: Vibrational spectroscopy is the most widely researched point spectroscopy method for characterization of normal and diseased tissue. It measures molecular vibrations, induced by incident light, corresponding to the chemical makeup at the molecular level in two different ways: absorption of electromagnetic radiation or frequency shifts between incident and scattered light—-the so-called Raman scattering effect.

In case of infrared (IR) absorption spectroscopy, the sample is irradiated with a mid-IR beam and the transmitted light is examined for absorption of energy. The absorption spectrum, a plot of absorption versus different wavelengths, can reveal the biochemical makeup of the molecules. IR spectroscopy has been used to analyze tissue constituents at a molecular level for almost 60 years [34], [35]. Indeed, IR spectra was investigated for characterization of normal and neoplastic tissue as far back as 1952 by Woernley [36], who also showed that the absorption at certain frequencies can be correlated with the concentrations of nucleic acids in tissues. Recent advances in machine learning and pattern recognition algorithms and the development of the IR spectroscopic imaging modality have renewed interest in this technique for studying the biochemical makeup of healthy and diseased tissue.

In Raman spectroscopy, the sample is illuminated with a monochromatic visible or near-IR (NIR) light from a laser source and frequency shifts in the scattered light are measured. The Raman spectrum is a plot of intensity of the scattered photon versus shifts in its frequency, often measured in terms of wave numbers in $\mathrm{cm}^{-1}$. NIR-Raman spectroscopy is often used as an alternative to IR spectroscopy since NIR light has higher energy than mid-IR light and can penetrate much farther into the sample.

Fourier-transform (FT) spectroscopy, known as FT-IR when IR light is used, allows a faster acquisition of the IR spectra by using an interferometer followed by the Fourier transform (FT). FT-IR spectroscopy is the most commonly used form of IR spectroscopy. 
2) Spectral Imaging: Spectral imaging is carried out by building an image cube with slices corresponding to images of the same scene obtained by incident light at differing wavelengths. This technique is referred to as multispectral (MS) or hyperspectral (HS) imaging depending on the number $N_{b}$ of spectral bands, individual slices of the image cube in the spectral direction (generally with $N_{b} \leq 40$ for MS and $N_{b}>40$ for HS).

3) Spectroscopic Imaging: Spectroscopic imaging is similar to spectral imaging in that a volumetric cube is obtained with a spectrum per pixel in the optical field. The main difference is that spectroscopic imaging builds the image cube by dividing the image scene into a uniform Cartesian grid, raster scanning the scene according to the grid, and collecting point spectra for each of the grid points.

Fernandez et al. [33] have proposed an IR spectroscopic imaging method based on a Michelson interferometer and all-reflecting microscope equipped with a 16-element linear array detector with a narrow aperture size of $6.25 \mu \mathrm{m} \times 6.25$ $\mu \mathrm{m}$. A massive 1641-dimensional point spectrum was obtained for each pixel spanning a spectral range of $4000-720 \mathrm{~cm}^{-1}$ at an effective spectral resolution of $2 \mathrm{~cm}^{-1}$ and at a spatial resolution of $6.25 \mu \mathrm{m}$. Tissue sections were also stained with $\mathrm{H} \& \mathrm{E}$ and imaged with a digital light microscope for manual histopathology analysis.

While most of the above methods are generally invasive for internal body organs, magnetic resonance spectroscopy (MRS) is a completely noninvasive way of probing the biochemical makeup of tissue. By this virtue, it is a particularly attractive prospect for the imaging of brain tumors, along with magnetic resonance (MR) imaging which has become a mainstay in the diagnosis of suspicious brain lesions [37]. The main principle behind MRS imaging is the chemical shift process, the process whereby different metabolites in the tissue respond at different resonating frequencies, with the chemical shift often measured in parts per million (ppm). One particular advantage of MRS is that it can be tuned to specific nuclei in the tissue; with hydrogen $\left({ }^{1} \mathrm{H}\right.$, also known as proton) being the most commonly studied one. Studies have shown clear differences between ${ }^{1} \mathrm{H}$ MRS spectra of brain tumors and normal brain [38].

4) Spectral Analysis for Histopathology: In IR spectroscopy, McIntosh et al. [39] investigated the use of infrared spectroscopy for the characterization of in vitro basal cell carcinoma (BCC) specimens, exploiting the fact that mid-IR light is absorbed by a variety of skin components. Point spectroscopy was performed using an IR spectrometer and an aperture of $20 \mu \mathrm{m} \times 20 \mu \mathrm{m}$ from carefully selected regions containing only one type of skin lesion. Their analysis of the normalized spectra employing linear discriminant analysis (LDA) identified absorption bands that arise mainly from $\mathrm{CH} 2$ and $\mathrm{CH} 3$ absorptions in dermal spectra that are similar to those seen in samples rich in protein and collagen in particular. H\&E staining for standard histological examination was carried out after the spectra had been obtained. In a more recent paper, McIntosh et al. [40] utilized LDA to analyze the near-IR (NIR) absorption spectrum for noninvasive, in vivo characterization of skin neoplasms. Their rationale for using NIR light was that the mid-IR light could be completely absorbed by samples greater than $10-15 \mu \mathrm{m}$ in thickness, therefore limiting the utility of mid-IR spectroscopy to in vitro analysis.

In Raman spectroscopy, Frank et al. [41] examined Raman spectra from breast and observed that visible laser excitation could be used to reveal Raman features for lipids and carotenoids. Huang et al. [42] explored the use of a rapid acquisition NIR Raman spectroscopy system for in vitro diagnosis of lung cancer. Student's $t$-test was performed to discriminate between normal and malignant bronchial tissues using the ratio of Raman intensity at two specific wavelengths. Chowdary et al. [43] showed that the Raman spectra could be useful for discriminating between normal and diseased breast tissues, although a simple principle component analysis (PCA) of spectra was employed for discrimination purposes. Analyzing the Raman spectra of malignant breast tissues, they concluded that malignant tissues had an excess of lipids and proteins. Robichaux-Viehoever et al. [44] investigated the use of NIR Raman spectra for the detection of cervical dysplasia and achieved high correlation between the results of their spectral analysis and the histopathology diagnosis.

Recently, Wang et al. [45] have shown that FT-IR spectroscopy can be effectively used for detecting premalignant (dysplastic) mucosa and leads to better inter-observer agreement, in terms of the $\kappa$-statistic. Oliveira et al. [46] have explored a setup involving a fixed-wavelength $(1064 \mathrm{~nm})$ laser line as an excitation source and FT-Raman for generating the spectra. Spectral analysis using PCA and Mahalanobis distance were used to detect dysplastic and malignant oral lesions. Their results using LDA showed effective separation of spectra of benign lesions from those of premalignant and malignant lesions.

Over the years, MS and HS imaging have demonstrated an enormous potential in remote-sensing applications, leading many researchers to expect promise about their usefulness in histopathology [47]. This promise has been demonstrated by and [48] in their work on the diagnosis of colon adenocarcinoma. However, two recent studies [49], [50] have found that the additional spectral information does not significantly improve the classification results. This may be due to the fact that most MS and HS imaging methods employ the visible part of light spectrum which may not be very useful in terms of highlighting important biochemical characteristics, as opposed to the near-IR or mid-IR spectrum. The number of stains present in the sample, as well as the characteristics of the stains themselves will also directly affect the performance of MS and HS image analysis methods.

In MRS, several studies, such as [51], can be found in the literature that report high correlation between automatic grading of in vivo tumors and their corresponding post-operative histopathology findings. However, MRS spectral analysis has traditionally been limited to rather simplistic ratio tests. Tiwari et al. [52] recently proposed an unsupervised spectral clustering-based algorithm for diagnosis of prostate cancer from the MRS spectra, reporting higher specificity compared to the popular $z$-score scheme, routinely used for the analysis of MRS data.

Spectral analysis using different modalities discussed above has demonstrated its potential for diagnosis and grading of 
cancer in tissue samples. However, as outlined above, most of the proposed algorithms in the literature make use of linear sub-space projection methods (PCA, LDA, etc.) for analysis of the spectral data despite the fact that spectral signatures of different types of tissue in the high-dimensional space may not be linearly separable. Furthermore, a few challenges are limiting the success of such methods in the realm of optical diagnostics. These include, but are not limited to, storage and transmission, registration, processing and analysis of large amounts of data generated by spectral data acquisition methods. The problem is further compounded when gathering spectral imaging data for whole slides. However, the availability of powerful computational resources at increasingly low prices and recent advances in image analysis have meant that more sophisticated methods can now be applied for analyzing large amounts of spectral data.

\section{IMAGE PREPROCESSING: COLOR AND ILlUMINATION NORMALIZATION}

\section{A. Color Normalization}

One of the first steps essential for both fluorescent and bright field microscopy image analysis is color and illumination normalization. This process reduces the differences in tissue samples due to variation in staining and scanning conditions. The illumination can be corrected either using calibration targets or estimating the illumination pattern from a series of images by fitting polynomial surfaces [29]. Another approach is to match the histograms of the images. Software that corrects for spectral and spatial illumination variations is becoming a standard package provided by most bright field manufacturers. This is an essential step for algorithms that heavily depend on color space computations. Yang and Foran [53] presented a robust color-based segmentation algorithm for histological structures that used image gradients estimated in the $L U V$ color space to deal with issues of stain variability. In the next section, we give detailed description of correcting another artifact, tissue autofluorescence, in fluorescent images.

\section{B. Compensating for Tissue Auto-Fluorescence}

Tissue auto-fluorescence (AF) is a fundamental problem in microscopy applications, particularly in retrospective studies that use formalin fixed paraffin embedded tissue sections. AF reduces the signal detection sensitivity, and in some cases even causes failure in the detection of fluorescent biomarker signals. In [29], a two-step technique was used to remove the $\mathrm{AF}$ from fluorescent microscopy images. Rather than acquiring images of all the dyes at once using a set of optimum filter cubes tuned to specific dyes, the acquisition is done in two steps. In the first step, tissue is stained with only the low AF dyes (e.g., ultraviolet or infrared), and images are acquired using all the filter cubes. Images of these cubes, except the low AF dyes, represent the tissue AF at their specific spectra. In the second step, all the remaining dyes are added, and images of all the cubes are acquired again. Then the first set of images is aligned with the second set using a transformation estimated by registering the low-AF images that are common in both steps.
The first step before any AF removal is the correction of the excitation light pattern. The observed image, $I(x, y)$, can be modeled as a product of the excitation pattern, and the emission pattern. While the emission pattern captures the tissue dependent fluorescent staining, the excitation pattern captures the excitation light. In the logarithm domain, the multiplicative relation can be transformed into a linear form. The excitation pattern can be estimated using the mean of the brightest set of pixels from an ordered set of $N$ images

$$
E^{\prime}{ }_{\mathrm{AVE}}(x, y)=\frac{1}{K} \sum_{n=1}^{K} \log \left(I_{n}(x, y)\right)
$$

where $I_{n}(x, y)$ denote the ordered pixels $\left(I_{1}(x, y) \geq\right.$ $\left.I_{2}(x, y) \geq \cdots I_{n}(x, y) \cdots \geq I_{N}(x, y)\right)$, and $K$ represents the set of brightest pixels. Assuming that a certain percentage of the image occupies stained tissue (nonzero backgound), $K$ is set to an integer to represent this percentage (10\% in our experiments). This approximation holds if a large number of images are used in the averaging process. However, a large percentage of pixels are already excluded to eliminate the nontissue pixels in the images. To overcome the limited sampling size, the log of the excitation pattern estimated in (3.1) can be approximated with polynomials. The surface generated by the polynomial coefficients are then used to correct individual images [54].

After the images are corrected for their light excitation pattern, the images between the two sequential steps are aligned. From the two common images, one being the reference image from the first step, $F(x, y)$, and the second being from the subsequent step, $S(x, y)$, a rigid transformation $\mathbf{T}_{F S}$ is obtained, such that the image similarity measure, between $F(x, y)$ and $S\left(\mathbf{T}_{F S}(x, y)\right)$ is maximized

$$
\arg \max _{\mathbf{T}_{F S}} \operatorname{Similarity}\left(F(x, y), S\left(\mathbf{T}_{F S}(x, y)\right)\right) .
$$

Due to its robustness in registering multimodality images, a mutual information-based image similarity measure is used to deal with tissue loss and folding. Additional robustness is achieved by incorporating the mutual information estimation in a multiresolution framework [55].

Once $\mathbf{T}_{F S}$ is estimated, all the channels are transformed with this transformation to represent all the images in both acquisitions in the same coordinate system. Then the first set of images is subtracted from the second set of images. To achieve the highest dynamic range, the first set of AF images can be acquired in longer exposure times than the second set of AF-dye mixture images. Fig. 3 shows the acquisition of the $\mathrm{Cy} 3$ channel before and after the tissue is stained with Cy3 dye directly conjugated with Estrogen Receptor (ER). The tissue is also stained with a low AF ultraviolet nuclear stain, DAPI (not shown in the figure), which is acquired in both steps and used for aligning the images. The AF removed image is shown in Fig. 3(c). The arrows point to successfully removed high-AF regions, such as red blood cells. Removing the AF using the proposed two-step approach enables accurate image analysis and quantitation for low abundance proteins and directly conjugated antibodies. 


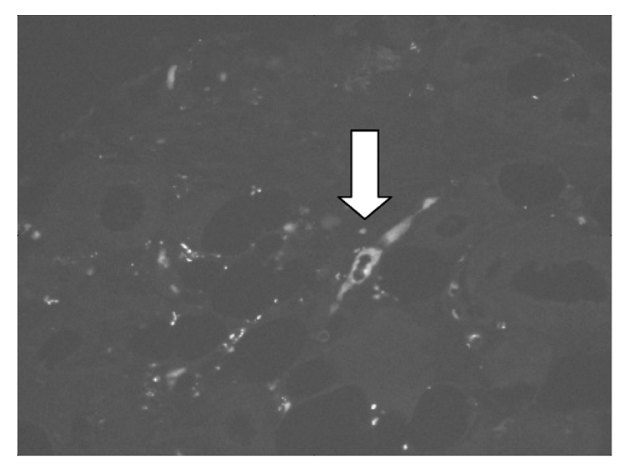

(a)

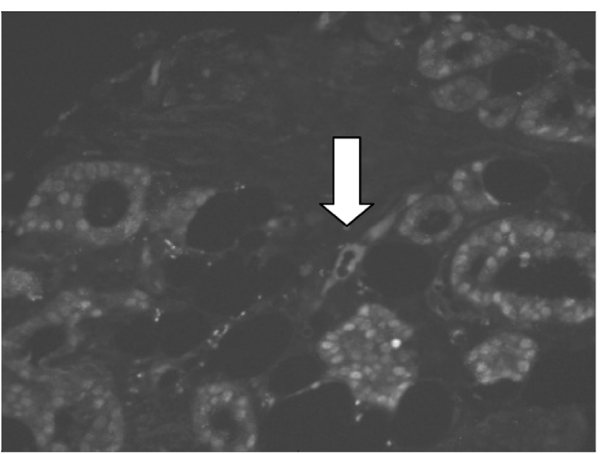

(c)

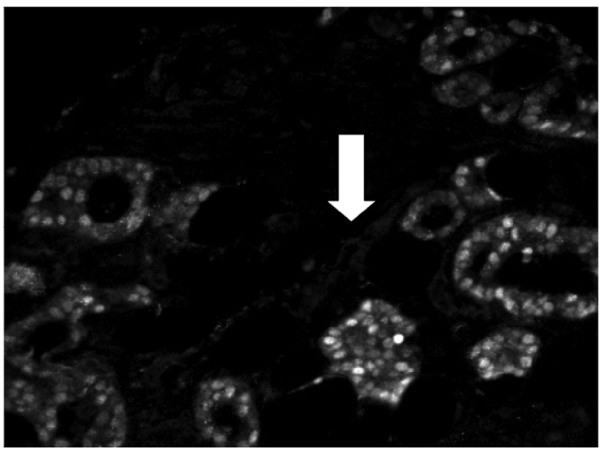

(e)

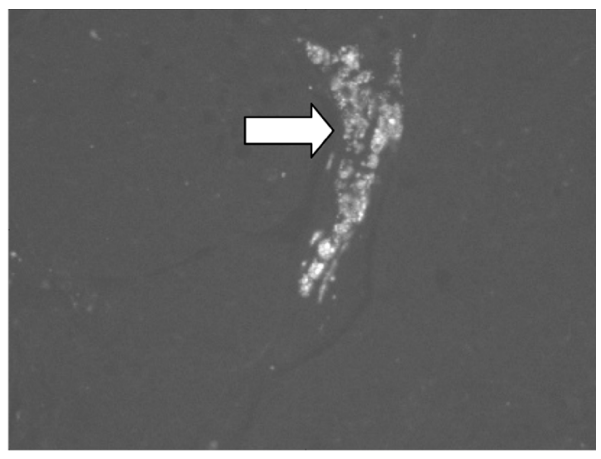

(b)

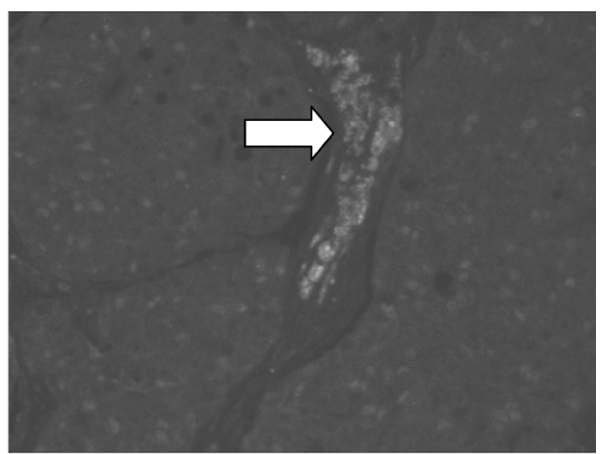

(d)

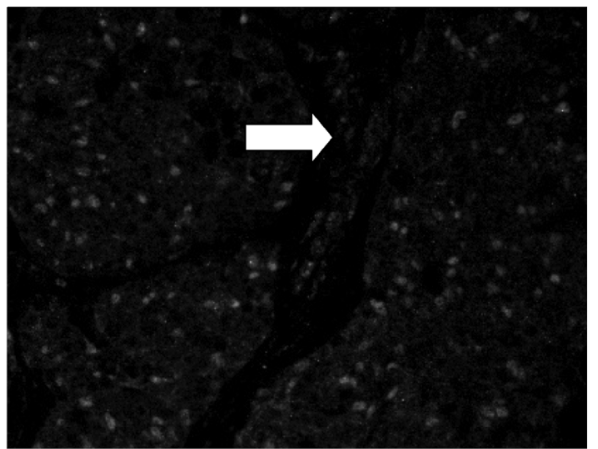

(f)

Fig. 3. (a), (b) Images from the first step acquisition. (c), (d) Images from the second acquisition. (e), (f) AF-free corrected images. Cy5 dye (a), (c), (e) is directly conjugated to Pan-Cadherin, a membrane protein. Cy3 dye (b), (d), (f) is directly conjugated to Estrogen Receptor. The arrows point to successfully removed the high-AF regions, such as blood cells and fat.

\section{Automated Detection AND SEgmentation OF HISTOPATHOLOGY IMAGES}

One of the prerequisites to grading or diagnosis of disease in histopathology images is often the identification of certain histological structures such as lymphocytes, cancer nuclei, and glands. The presence, extent, size, shape, and other morphological appearance of these structures are important indicators for presence or severity of disease. For instance, the size of the glands in prostate cancer tend to reduce with higher Gleason patterns [56]. Similarly, the presence of a large number of lymphocytes in breast cancer histopathology is strongly suggestive of poor disease outcome and survival [57]. Consequently, a prerequisite to identification and classification of disease is the ability to automatically identify these structures. These approaches can either be global, in which they attempt to simultaneously segment all the structures in the image scene or local approaches which target specific structures.

Another motivation for detecting and segmenting histological structures has to do with the need for counting of objects, generally cells or cell nuclei. Cell counts can have diagnostic significance for some cancerous conditions. Bibbo et al. [58] reported $1.1 \%-4.7 \%$ error in cell counts compared to manual counts for Feulgen-stained prostate specimens. Belien et al. [59] found $19 \%-42 \%$ error in counting mitoses in Feulgen-stained breast tissue sections. In immunohistochemically stained bone marrow biopsies, Markiewicz et al. [60] reported 2.8\%-10.0\% difference in counts between manual and automatic methods, while Kim et al. [61] found a correlation of 0.98 between manual and automatic counts of immunostained slides of meningiomas. Sont et al. [62] found a correlation of 0.98 between automated and semi-automated methods for inflammatory cell counts in immunostained bronchial tissue. 


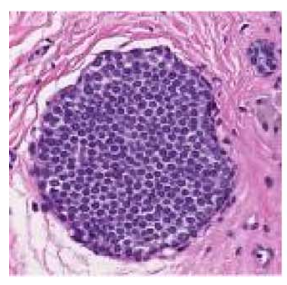

(a)

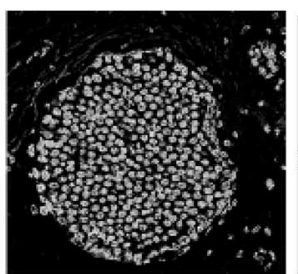

(b)

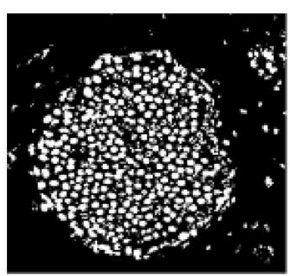

(c)

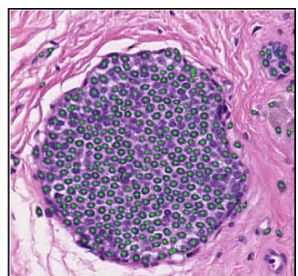

(d)

Fig. 4. (a) Original DCIS image with corresponding (b) likelihood scene obtained via a Bayesian classifier driven by color and texture. (c) Thresholded version of likelihood scene ( $95 \%$ confidence). (d) The final nuclear segmentation obtained by integrating the Bayesian classifier with the template matching scheme.

\section{A. Local, Structural Segmentation}

1) Nuclear Segmentation: Numerous works have been conducted [63]-[65] on segmentation of various structures in breast histopathology images using methodologies such as thresholding, fuzzy c-means clustering, and adaptive thresholding [65]. Thresholding tends to work only on uniform images and does not produce consistent results if there is considerable variability within image sets. Watershed algorithms tend to pose the same problem [64] due to variability in image sets. Active contours are widely used in image segmentation; however, contours enclosing multiple overlapping objects pose a major limitation. In addition, inclusion of other irrelevant objects from the background further complicates the possibility of obtaining a viable segmentation.

The pixel-level analysis of unstained prostate slides by Fourier transform infrared spectroscopy resulted in 94\%-100\% accuracy in the pixel-level classification of ten histologic classes as reported by Fernandez et al. in [66]. The pixel-level classification of nuclear material by Boucheron et al. [67] resulted in performances (equal tradeoff between detection and false alarm rates) of $88 \%-90 \%$ for H\&E stained breast tissue. The use of automated methods for pixel-level analysis is perhaps more common for immunostained or fluorescently stained specimens. Singh et al. [68] reported 98\% accuracy in the detection of positive and negative prostate nuclei immunostained for androgen receptor protein expression. Analysis of cytokeratin-stained lymph node sections yielded $95 \%$ detection of stained cells as reported by Weaver et al. in [69]. However, these studies focus only on finding nuclei on a pixel level.

In H\&E stained imagery of astrocytomas and bladder tissue, Glotsos et al. [70] reported that $94 \%$ of nuclei were correctly delineated. Latson et al. found $25 \%$ poorly segmented nuclei, $4.5 \%-16.7 \%$ clumped nuclei, and $0.4 \%-1.5 \%$ missed nuclei in $\mathrm{H} \& \mathrm{E}$ stained breast biopsies. Fluorescently stained imagery of cervical and prostate carcinomas allowed for $91 \%-96 \%$ accuracy in cell segmentation by Wahlby et al. [71], where the accuracy here is calculated based on manual cell counts (i.e., not taking into account the accuracy of the actual nuclear delineation). Korde et al. used image intensity thresholding to segment nuclei in the bladder and in skin tissue [72]. Gurcan et al. leveraged gray level morphology followed by hysteresis thresholding to achieve cell nuclei segmentation in digitized H\&E stained slides [73], [74]. Other algorithms have been proposed using more complex techniques, such an active contour scheme for pap-stained cervical cell images by Bamford and Lovell [75] and a fuzzy logic engine proposed by Begelman et al. [76] for prostate tissue that uses both color- and shape-based constraints.

In [63] and [77], nuclear segmentation from breast and prostate cancer histopathology was achieved by integrating a Bayesian classifier driven by image color and image texture and a shape-based template matching algorithm (Fig. 4). Fig. 4(a) shows a Ductal carcinoma in situ (DCIS) study with a number of nuclei closely packed together. The likelihood image representing the probability of each pixel corresponding to a nuclear region is shown in Fig. 4(b). Note that several nuclei lie adjacent to each other and hence template matching is used to extricate the individual nuclei. Fig. 4(c) shows the result of thresholding the Bayesian likelihood scene $(95 \%$ confidence level). Template matching is then done at every location in Fig. 4(c). Only those image locations where correspondence between the binary segmentation [Fig. 4(c)] and the template was found are shown as bright. The final nuclear boundary detection (green dots) is displayed in Fig. 4(d).

2) Gland Segmentation: In a recently presented scheme for extracting glandular boundaries from histopathology scenes [63], the algorithm consists of three distinct components: In the first stage, a Bayesian classifier is trained based on color and textural information to automatically identify nuclei, cytoplasm, and lumen regions in the scene. Following low-level Bayesian classification, structural constraints are incorporated to constrain the segmentation by using image information regarding the specific order of arrangement of glandular structures (central lumen, surrounding cytoplasm, and nuclear periphery) in order to reduce the number of false positive gland regions. Finally, a shape-based segmentation method in the form of level sets [78] is initialized within candidate lumen regions as determined from the Bayesian classifier. Hence, the level set surface evolution is controlled by the Bayesian probability scene derived via use of the low-level image information. The level set evolution is stopped at the interface between lumen and cytoplasm and, thus, a segmentation of the inner gland boundary is obtained. A second level set is then initialized within the cytoplasm area and used to capture the outer gland margin. Once the possible gland lumens are found, boundary segmentation is performed using level-sets. A boundary $\mathrm{B}$ evolving in time $\mathrm{t}$ and in the 2-D space defined by the grid of pixels $\mathrm{C}$ is represented by the zero level set $\mathrm{B}=\{(\mathrm{x}, \mathrm{y}) \mid \mathrm{f}(\mathrm{t}, \mathrm{x}, \mathrm{y})=0\}$ of a level set function $\mathrm{f}$, where $\mathrm{x}$ 

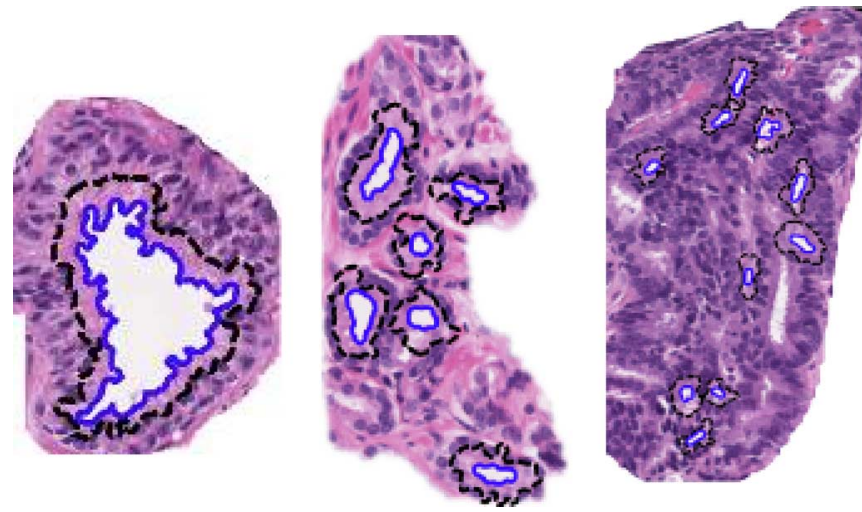

Fig. 5. Results of the automatic segmentation algorithm (blue contours: lumen boundary, black contours: inner boundary of the nuclei of the epithelial cells surrounding the gland). Shown from left to right are example images of benign epithelium, intermediate-, and high-grade cancer.

and $\mathrm{y}$ are 2-D Cartesian coordinates of $\mathrm{c} \in \mathrm{C}$. The evolution of $\mathrm{f}$ is then described by a level-set formulation adopted from [78]

$$
\frac{\partial \phi}{\partial t}+F|\nabla \phi|=0
$$

where the function $F$ defines the speed of the evolution. The curve evolution is driven by the nuclei likelihood image. The initial contour $\phi_{0}=\phi(0, x, y)$ is initialized automatically using the detected lumen area from the candidate gland regions. The curve is evolved outward from the detected lumen regions in the combined nuclei likelihood image to avoid noise and allow smoother evolution relative to the original image. The intensities of the nuclei likelihood image form the stopping gradient. The algorithm is run until the difference in the contours in two consecutive iterations is below an empirically determined threshold. During training, size distributions similar to those used to calculate object likelihood are created using the final contours. These nuclear boundary based distributions are used to remove regions that are too large to be true glands. Finally, the lumen and nuclear boundaries extracted from true gland regions are passed on to the next step for feature extraction. Sample results from the automated gland segmentation algorithm are shown in Fig. 5. The lumen boundaries are displayed in a solid blue contour and the interior nuclear boundaries are displayed as dashed black lines. Results of our gland segmentation algorithm are shown for sample images from benign epithelium, intermediate, and high-grade prostate cancer (from left to right).

\section{B. Global Scene Segmentation Approaches}

In [26], a unified segmentation algorithm for sub-cellular compartmentalization was presented. Quantitation of biomarkers at sub-cellular resolution requires segmentation of sub-cellular compartments such as nuclei, membranes, and cytoplasm. While different segmentation algorithms can be used for each of the sub-cellular compartments, an alternative is to use the same algorithm in different modes. The algorithm in [26] captured a set of bright pixels sharing a common shape distribution. The algorithm used a set of three features, one is the fluorescent emission intensity, and the other two are based on curvature descriptors that are computed from the eigenvalues of the Hessian matrix.

For an image, $I(x, y)$, the eigenvalues $\left(\lambda_{1}(x, y) \leq \lambda_{2}(x, y)\right)$ of the Hessian matrix encode the curvature information of the image, and provide useful cues for detecting ridge-like membrane structures, or blob-like nuclei structures. However, the eigenvalues are dependent on image brightness. The following two curvature-based features are independent of image brightness:

$$
\begin{aligned}
\theta(x, y) & =\operatorname{atan} 2\left(\lambda_{1}(x, y), \lambda_{2}(x, y)\right) \\
p h i(x, y) & =\tan ^{-1} \frac{\left(\lambda_{1}(x, y)^{2}+\lambda_{2}(x, y)^{2}\right)^{1 / 2}}{I(x, y)}
\end{aligned}
$$

and referred to as shape index, and normalized-curvature index, respectively. This is essentially the same as defining the eigenvalues in a polar coordinate system. This transformation also results in bounded features, $-3 \pi / 4 \leq \theta(x, y) \leq \pi / 4$, and $0 \leq \phi(x, y) \leq \pi / 2$.

The estimation process starts with the expected distributions of the shape index for the structures to be segmented. For example, for bright membrane and vessel like structures the shape index is close to $-\pi / 2$, because the smaller eigenvalue is negative and the larger eigenvalue approaches to zero. On the other hand, for the blob-like nuclei structures, the shape index is close to $-3 \pi / 4$, because both eigenvalues are negative and close in value. For both structures, positive values indicate a pixel being more like a background. These constraints are used to compute the initial foreground and background sets for membrane and nuclei structures. An initial segmentation based on the shape index and the normalized-curvature index separates the image pixels into three subsets: background, foreground, and indeterminate. The indeterminate subset comprises all the pixels that are not included in the background or foreground subsets. From these subsets, the background and foreground intensity distributions, as well as the intensity log-likelihood functions are estimated. The algorithm keeps iterating by using two out of the three features at a time to estimate the distribution of the feature that is left out. In the final step, these log-likelihood functions are combined to determine the overall likelihood function. A probability map that represents the probability of a pixel being a foreground is calculated.

Cytoplasm can be detected either by using a specific cytoplasmic marker, or can be detected using computational methods using the fact that the cytoplasmic areas are between nuclear and membrane areas. For most cancer tissue types, it is very important to differentiate the epithelial tissue from the stromal and connective tissue, so that for IHC studies the expression levels of most markers in the epithelial regions can be quantified. Computational methods that use the high connectivity of membrane meshes can be used to differentiate the epithelial regions. For the sample images, any connected component larger than 800 pixels is accepted as a part of the epithelial mask. The nuclei set is then separated into epithelial nuclei and stromal nuclei using the epithelial mask. 
TABLE I

Summary of OBJeCt-LeVEl Features USED IN Histopathology IMAGE ANALYSIS

\begin{tabular}{|c|c|}
\hline Category & Features \\
\hline \multirow[t]{9}{*}{ Size and Shape } & Area \\
\hline & $\begin{array}{l}\text { Elliptical Features: Major and minor axis length, eccentricity, } \\
\text { orientation, elliptical deviation }\end{array}$ \\
\hline & Convex Hull Features: Convex area, convex deficiency, solidity \\
\hline & Filled Image Features: Filled area, Euler number \\
\hline & Bounding Box Features: Extent, aspect ratio \\
\hline & $\begin{array}{l}\text { Boundary Features: Perimeter, radii, perimeter Fourier energies, } \\
\text { perimeter curvature, bending energy, perimeter fractal dimension }\end{array}$ \\
\hline & $\begin{array}{l}\text { Other Shape Features: Equivalent diameter, sphericity, } \\
\text { compactness, inertia shape }\end{array}$ \\
\hline & Center of Mass \\
\hline & Reflection Symmetry \\
\hline \multirow{3}{*}{$\begin{array}{l}\text { Radiometric and } \\
\text { Densitometric }\end{array}$} & Image Bands, Intensity \\
\hline & Optical density, integrated optical density, and mean optical \\
\hline & Hue \\
\hline \multirow[t]{5}{*}{ Texture } & $\begin{array}{l}\text { Co-occurrence Matrix Features: Inertia, energy, entropy, } \\
\text { homogeneity, maximum probability, cluster shade, cluster }\end{array}$ \\
\hline & Fractal Dimension \\
\hline & $\begin{array}{l}\text { Run-length Features: Short runs emphasis, long runs emphasis, } \\
\text { gray-level non-uniformity, run-length non-uniformity, runs } \\
\text { percentaqe. low qrav-level runs emphasis. hiqh qrav-level runs }\end{array}$ \\
\hline & Wavelet Features: Energies of detail and low resolution images \\
\hline & Entropy \\
\hline $\begin{array}{l}\text { Chromatin- } \\
\text { Specific }\end{array}$ & $\begin{array}{l}\text { Area, integrated optical density, mean optical density, number of } \\
\text { regions, compactness, distance, center of mass }\end{array}$ \\
\hline
\end{tabular}

EMLDA is an image segmentation method, which uses the Fisher-Rao criterion as the kernel of the expectation maximization (EM) algorithm [79]. Typically, the EM-algorithm is used to estimate the parameters of some parameterized distributions, such as the popular Gaussian mixture models, and assign labels to data in an iterative way. Instead, the EMLDA algorithm uses the linear discriminant analysis (LDA), a supervised classification technique, as the kernel of EM-algorithm and iteratively group data points projected to a reduced dimensional feature space in such a way that the separability across all classes is maximized. In [62], the authors successfully applied this approach in the context of histopathological image analysis to achieve the segmentation of digitized $\mathrm{H} \& \mathrm{E}$ stained whole-slide tissue samples.

\section{Feature Extraction}

Research on useful features for disease classification has often been inspired by visual attributes defined by clinicians as particularly important for disease grading and diagnosis. The vast majority of these features are nuclear features and many have been established as useful in analysis of both cytopathology and histopathology imagery. Other features that assume discriminatory importance include the margin and boundary appearance of ductal, stromal, tubular, and glandular structures. While there is a compilation of features for cytopathology imagery [80], there is relatively little such work for histopathology imagery.

Humans' concept of the world is inherently object-based, as opposed to the largely pixel-based representation of computer vision. As such, human experts describe and understand images in terms of such objects. For pathologists, diagnosis criteria are inevitably described using terms such as "nucleus" and "cell." It is thus important to develop computer vision methods capable of such object-level analysis.

\section{A. Object Level Features}

Fundamentally, object-level analysis depends greatly on some underlying segmentation mechanism. It is the segmentation methodology that determines what constitutes an object. Commonly, an object is defined as a connected group of pixels satisfying some similarity criterion. The main focus is often on the segmentation of nuclei; there exists little work that explicitly uses features of cytoplasm and stroma, although some researchers have hinted at the need for such features [81], [82]. Preliminary work [83] has demonstrated the feasibility of other histologic features for image classification in H\&E stained breast cancer. Madabhushi et al. [63] used cytoplasmic and stromal features to automatically segment glands in prostate histopathology. Moreover, it appears that histologic objects may not need to be perfectly segmented to be properly classified when a list of comprehensive features is used in a feature selection framework [83]. Classification performance in distinguishing between different grades of prostate cancer was found to be comparable using manual and automated gland and nuclear segmentation [63]. These results suggest that perfect segmentation is not necessarily a prerequisite for good classification.

Object-level features can be categorized as belonging to one of four categories: size and shape, radiometric and densitometric, texture, and chromatin-specific. While the radiometric and densitometric, texture, and chromatin-specific features could be considered low-level features that can be extracted from local neighborhoods, the size and shape metrics are true object-level metrics. A summary of object-level features is listed in Table I; definitions for all listed features can be found in [83]. These features were compiled from a comprehensive literature search on cytopathology and histopathology image analysis. In addition, various statistics measures for any of 
the vector quantities are also commonly calculated. Thus, the mean, median, minimum, maximum, standard deviation, skewness, and kurtosis can be calculated for all vector features. For an RGB image, all relevant features are extracted for each individual color channel; hence, the total number of object-level features can easily exceed 1000 for the list of features in Table I. It should be noted that these features are most commonly extracted from high-resolution imagery (see next section), but are relevant for any resolution.

An approach that semantically describes histopathology images using model based intermediate representation (MBIR) and incorporates low-level color texture analysis was presented in [84]. In this approach, basic cytological components in the image are first identified using an unsupervised clustering in the $\mathrm{La}^{*} \mathrm{~b} *$ color space. The connected components of nuclei and cytoplasm regions were modeled using ellipses. An extensive set of features can be constructed from this intermediate representation to characterize the tissue morphology as well as tissue topology. Using this representation, the relative amount and spatial distribution of these cytological components can be measured. In the application of follicular lymphoma grading, where the spatial distribution of these regions varies considerably between different histological grades, MBIR provides a convenient way to quantify the corresponding observations. Additionally, low-level color texture features are extracted using the co-occurrence statistics of the color information. Due to the staining of the tissue samples, the resulting digitized images have considerably limited dynamic ranges in the color spectrum. Taking this fact into account, a nonlinear color quantization using self-organizing maps (SOM) is used to adaptively model the color content of microscopic tissue images. The quantized image is used to construct the co-occurrence matrix from which low-level color texture features are extracted. By combining the statistical features constructed from the MBIR with the low-level color texture features, the classification performance of the system can be improved significantly.

Fig. 6 shows some of the textural image features for discriminating between benign breast epithelial tissue [77] (DCIS, Fig. 6(a)) and DCIS (Fig. 6(d)). Fig. 6(b), (e), shows the corresponding Gabor filter responses while Fig. 6(c), (f) shows the corresponding Haralick feature images.

\section{B. Spatially Related Features}

Graphs are efficient data structures to represent spatial data and an effective way to represent structural information by defining a large set of topological features. Formally, a simple graph $G=(V, E)$ is an undirected and unweighted graph without self-loops, with $V$ and $E$ being the node and edge set of graph $G$, respectively.

Application of graph theory to other problem domains is impressive. Real-world graphs of varying types and scales have been extensively investigated in technological [85], social [86] and biological systems [87]. In spite of their different domains, such self-organizing structures unexpectedly exhibit common classes of descriptive spatial (topological) features. These features are quantified by definition of computable metrics.

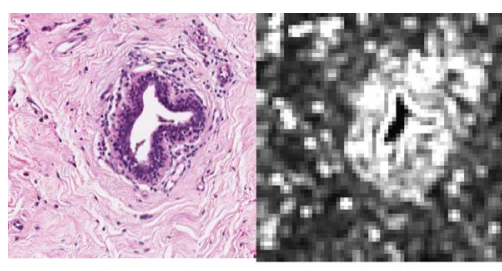

(a)

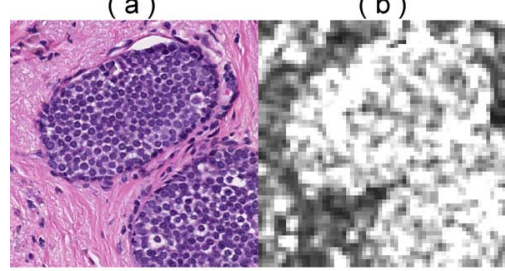

( d ) (e)

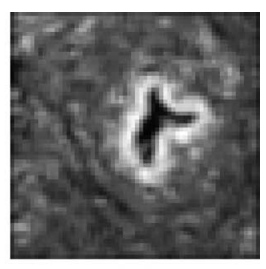

(c)

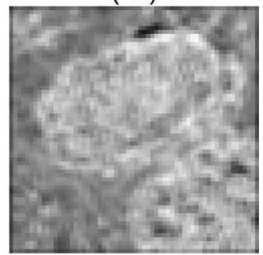

( $f$ )
Fig. 6. Supervised extraction of histological features to describe tissue appearance of (a) benign epithelium, and (b) DCIS. Feature images for the two tissue classes (benign epithelium, DCIS) corresponding to Gabor wavelet features (b), (e) and Haralick second order features (c), (f) are shown.
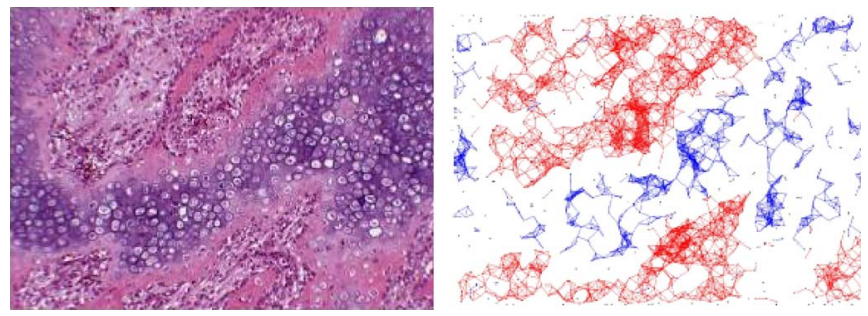

Fig. 7. Bone fracture and its corresponding ECM-aware cell-graph representation. Note the presence of a link between a pair of nodes in an ECM-aware cell-graph indicates not only topological closeness but also it implies the similarity in the surrounding ECM [90].

The use of spatial-relation features for quantifying cellular arrangement was proposed in the early 1990s [88], but did not find application to clinical imagery until recently. Graphs have now been constructed for modeling different tissue states and to distinguish one state from another by computing metrics on these graphs and classifying their values. Overall, however, the use of spatial arrangement of histological entities (generally at low resolutions) is relatively new, especially in comparison to the wealth of research on nuclear features (at higher resolutions) that has occurred during the same timeframe. A compilation of all the spatial-relation features published in the literature is summarized in Table II. Definitions for all graph structures and features can be found in [83]. The total number of spatial-relation features extracted is approximately 150 for all graph structures.

Graph theoretical metrics that can be defined and computed on a cell-graph induce a rich set of descriptive features that can be used for tissue classification. These features provide structural information to describe the tissue organization such as: i) the distribution of local information around a single cell cluster (e.g., degree, clustering coefficient); ii) the distribution of global information around a single cell cluster (e.g., eccentricity, closeness, between-ness); iii) the global connectivity information of a graph (e.g., ratio of the giant connected component over the graph size, percentage of the isolated and end data points in the graph); iv) the properties extracted from the spectral graph 
TABLE II

Summary of Spatial-ARRANGEMENT FEATURES USEd IN Histopathology IMAGE ANALYSiS.

\begin{tabular}{|l|l|}
\hline Graph Structure & Features \\
\hline Voronoi Tesselation & $\begin{array}{l}\text { Number of nodes, number of edges, cyclomatic number, } \\
\text { number of triangles, number of k-walks, spectral radius, } \\
\text { eigenexponent, Randic index, area, roundness factor, area } \\
\text { disorder, roundness factor homogeneity }\end{array}$ \\
\hline $\begin{array}{l}\text { Delaunay } \\
\text { Triangulation }\end{array}$ & $\begin{array}{l}\text { Number of nodes, edge length, degree, number of edges, } \\
\text { cyclomatic number, number of triangles, number of k-walks, } \\
\text { spectral radius, eigenexponent, Wiener index, eccentricity, } \\
\text { Randic index, fractal dimension }\end{array}$ \\
\hline $\begin{array}{l}\text { Minimum Spanning } \\
\text { Tree }\end{array}$ & $\begin{array}{l}\text { Number of nodes, edge length, degree, number of } \\
\text { neighbors, Wiener index, eccentricity, Randic index, } \\
\text { Balaban index, fractal dimension }\end{array}$ \\
\hline $\begin{array}{l}\text { O'Callaghan } \\
\text { Graph }\end{array}$ & $\begin{array}{l}\text { Number of nodes, number of edges, cyclomatic number, } \\
\text { number of neighbors, number of triangles, number of k- } \\
\text { walks, spectral radius, eigenexponent, Randic index, fractal } \\
\text { dimension }\end{array}$ \\
\hline Connected Graph & $\begin{array}{l}\text { Number of nodes, edge length, number of triangles, number } \\
\text { of k-walks, spectral radius, eigenexponent, Wiener index, } \\
\text { eccentricity, Randic index, fractal dimension }\end{array}$ \\
\hline $\begin{array}{l}\text { Relative Neighbor } \\
\text { Graph }\end{array}$ & $\begin{array}{l}\text { Number of nodes, number of edges, cyclomatic number, } \\
\text { number of neighbors, number of triangles, number of k- } \\
\text { walks, spectral radius, eigenexponent, Randic index, fractal } \\
\text { dimension }\end{array}$ \\
\hline k-NN Graph & $\begin{array}{l}\text { Number of nodes, edge length, degree, number of triangles, } \\
\text { number of k-walks, spectral radius, eigenexponent, Wiener } \\
\text { index, eccentricity, Randic index, fractal dimension }\end{array}$ \\
\hline
\end{tabular}

theory (e.g., spectral radius, eigen exponent, number of connected components, sum of the eigenvalues in the spectrum). Refer to Table II for a list of commonly extracted graph features.

1) 2-D Cell-Graph Construction: In cell-graph generation as proposed in [89], there are three steps: i) color quantization; ii) node identification; and iii) edge establishment. In the first step, the pixels belonging to cells from those of the others are distinguished. These steps are explained in the next sub-sections.

Node Identification: The class information of the pixels is translated to the node information of a cell-graph. At the end of this step, the spatial information of the cells is translated to their locations in the two-dimensional grid. After computing the probabilities, these are compared against a threshold value.

Edge Establishment: This step aims to model pair-wise relationships between cells by assigning an edge between them. Cells that are in physical contact are considered to be in communication, thus edges can be established between them deterministically. For other node pairs, a probability function is used to establish edges between a pair of nodes randomly. Since structural properties of different tissues (e.g., breast, bone and brain) are quite different from each other, edge establishment must be guided by biological hypothesis.

2) 3-D Cell-Graphs: The first step in 3-D cell-graph construction is to define the distance between a pair of nodes, which is simply the 3-D Euclidean distance between a pair of nodes. Based on this distance definition, edges can be established between a pair of nodes. In addition to the simple spatial distance metrics, a multidimensional distance measure can be defined using the cell-level attributes that can be provided by sophisticated image analysis and segmentation. Cell-level attributes include: $\mathrm{x}, \mathrm{y}, \mathrm{z}$ physical contact, volume with respect to number of pixels, peripheral (i.e., surface area), shared border as percentage of shared voxels relative to total, and polarity. Then each node of the 3-D cell-graph can be represented by a vector of $\mathrm{v}$-dimensions, each dimension corresponding to an attribute. The $L p$ norm can be used to compute the multidimensional distance between them. Once the notion of distance is determined, edge functions of cell-graphs can be applied to construct 3-D cell-graphs. The mathematical properties of cell-graphs in 3-D can be calculated as the feature set. Although most of the features defined on 2-D cell-graphs can be extended to the 3-D case, their calculation is not trivial.

\section{3) Application of Graph-Based Modeling for Different} Histopathology Related Applications:

Graph-Based Modeling of Extra Cellular Matrix: The extra cellular matrix (ECM) is composed of a complex network of proteins and oligosaccharides that play important roles in cellular activities such as division, motility, adhesion, and differentiation. Recently, a new technique was introduced for constructing ECM-aware cell-graphs that incorporates the ECM information surrounding the cells [90]. ECM-aware cell-graphs aim to preserve the interaction between cells and their surrounding ECM while modeling and classifying the tissues. The ECM-aware cell-graphs successfully distinguish between different types of cells that co-exist in the same tissue sample. For example, in bone tissue samples there are usually several cell types, including blood cells, normal cells, and sometimes fracture cells (e.g., chondrocytes and osteoblasts) and cancerous cells. Since these cells are functionally different from each other, the hypothesis is that they would exhibit different spatial organization and structural relationships in the same tissue. This hypothesis has been validated by showing that ECM-aware cell-graphs yield better classification results 


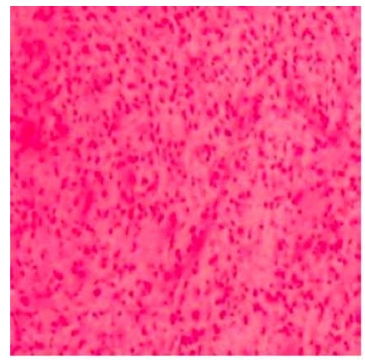

(a)

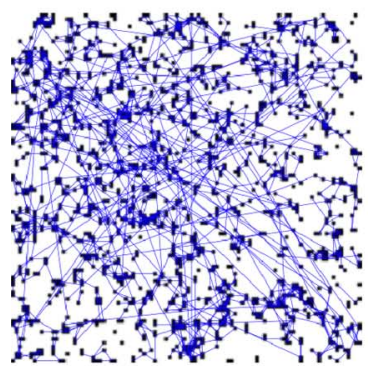

(d)

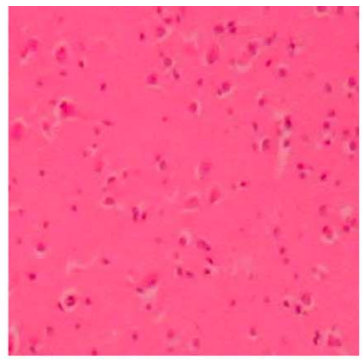

(b)

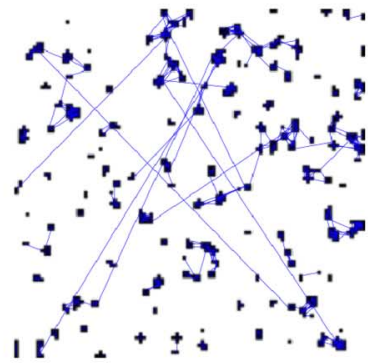

(e)

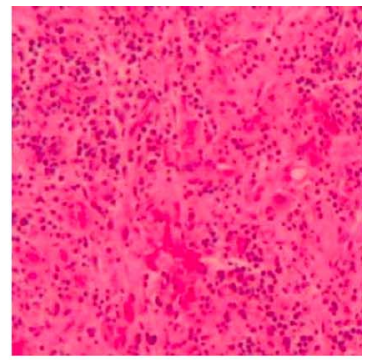

(c)

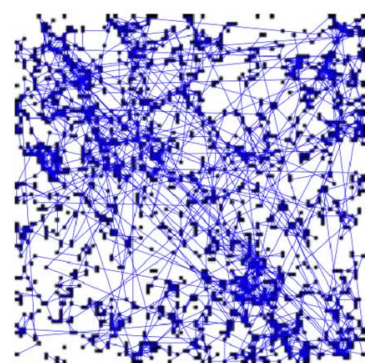

(f)

Fig. 8. Illustrating the differences between cell-graphs for cancerous, healthy, and inflamed brain tissues. Panels (a)-(c) show brain tissue samples that are (a) cancerous (gliomas), (b) healthy, and (c) inflamed, but noncancerous. Panels (d)-(f) show the cell-graphs corresponding to each tissue image. While the number of cancerous and inflamed tissue samples appear to have similar numbers and distributions of cells, the structure of their resulting cell-graphs shown in (d) and (f) are dramatically different. (Figure is taken from [91]).

for different states of bone tissues than the current state of art. In the construction a color value is assigned to each cell (i.e., vertex) based on the RGB values of its surrounding ECM. This is done by examining the $k$ neighboring pixels in each direction, and computing a dominant color for the ECM surrounding each cell using the RGB values of nearly $4 k^{2}$ neighboring pixels.

Application to Discriminating Different States of Brain Tissue: Fig. 8 shows the cell-graphs of brain tissues exhibiting distinctive graph properties that enable discrimination between the different states of brain tissue.

Application to Studying Temporal Activity of Adult Human Mesenchymal Stems Cells in a 3-D Collagen Matrix: Fig. 9 shows relationships between adult human mesenchymal stem cells in a 3-D collagen protein matrix over time in culture [90]. The graphs are generated from 3-D sections of tissue $(900 \times 900 \times 800 \mu \mathrm{m})$ imaged using confocal microscopy. The nuclei of stem cells in the constructs were stained and imaged at the time points indicated (0-24 h).

Application of Graph Theory to Modeling Cancer Grade: In [92], the Voronoi diagram is constructed from a set of seedlike points that denote the centers of each structure of interest (nuclei). From the Voronoi diagram, two more graphs of interest can be constructed: the Delaunay triangulation, which is created by connecting points that share an edge in the Voronoi diagram, and the minimum spanning tree, which is the series of lines that spans the set of points such that the Euclidean sum of the lengths of the lines is smaller than any other spanning tree. From each of these three graphs, a series of features are calculated that captures the size, shape, and arrangement of the structures of the nuclei. The graph-based representations of a Gleason grade 4 prostate histopathology image are shown in Fig. 10.

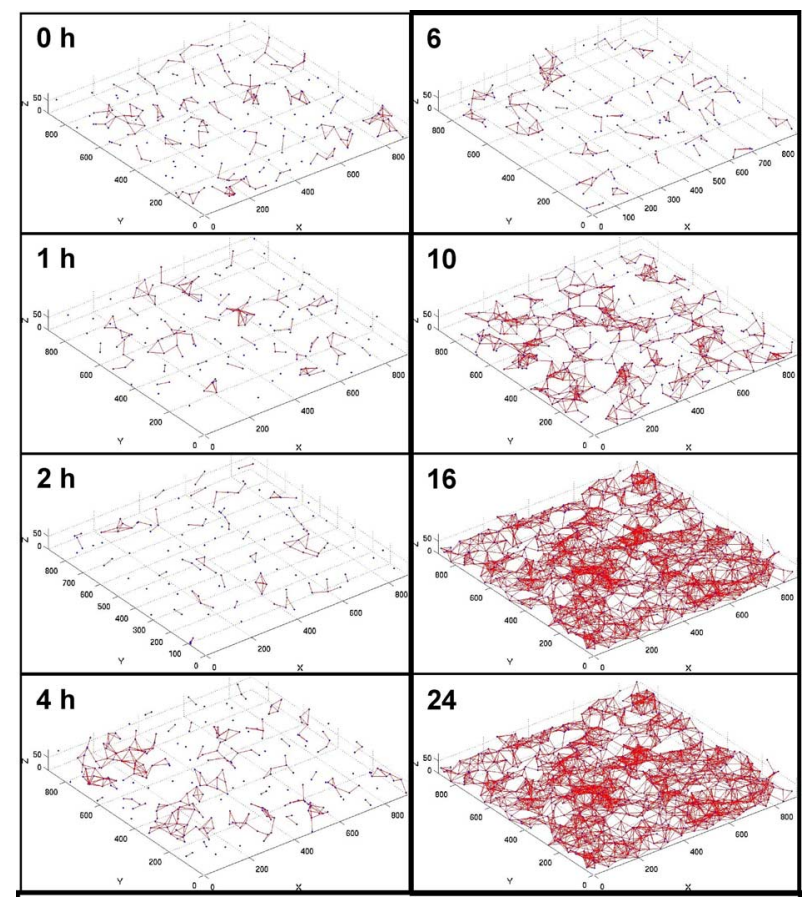

Fig. 9. Cell graphs produced from human MSC embedded in 3-D collagen matrices. Graphs show nuclei and development of edges (relationships) between them over time [90]. There is a phase transition sometime between hour 10 and hour 16 and the graph becomes connected.

\section{Multiscale Feature Extraction}

Owing to the density of the data and the fact that pathologists tend to employ a multiresolution approach to analyzing pathology data, feature values are related to the viewing scale 


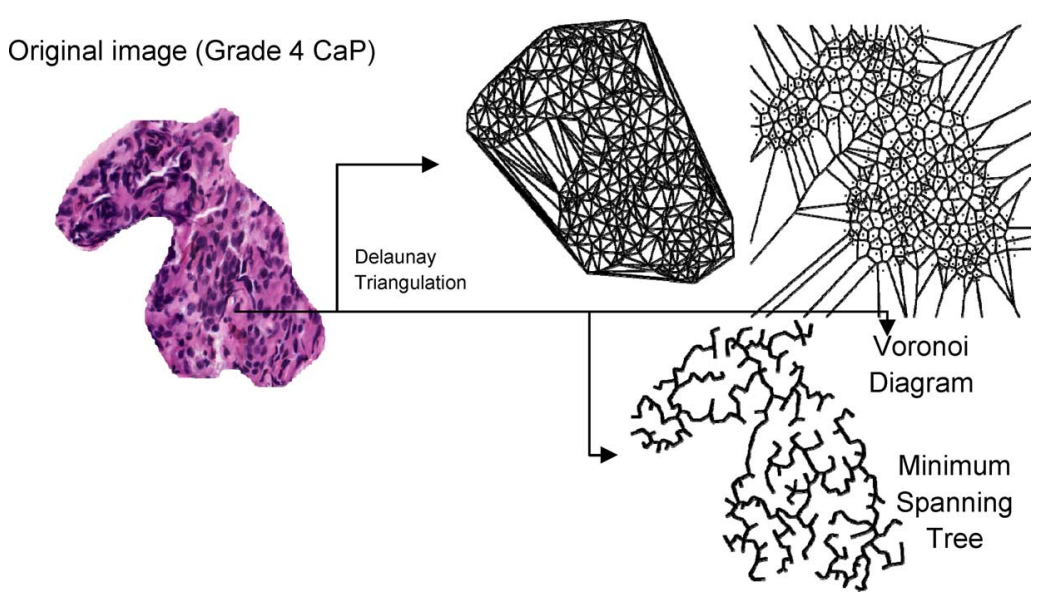

Fig. 10. (a) A digitized histopathology image of Grade $4 \mathrm{CaP}$ and different graph-based representations of tissue architecture via Delaunay Triangulation, Voronoi Diagram, and Minimum Spanning tree.

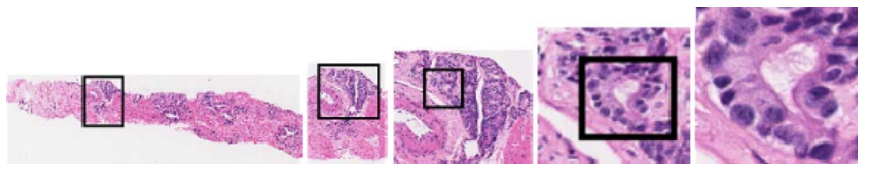

Fig. 11. Digitized histological image at successively higher scales (magnifications) yields incrementally more discriminatory information in order to detect suspicious regions.

or resolution. For instance at low or coarse scales color or texture cues are commonly used and at medium scales architectural arrangement of individual histological structures (glands and nuclei) start to become resolvable. It is only at higher resolutions that morphology of specific histological structures can be discerned.

In [93], [94], a multiresolution approach has been used for the classification of high-resolution whole-slide histopathology images. The proposed multiresolution approach mimics the evaluation of a pathologist such that image analysis starts from the lowest resolution, which corresponds to the lower magnification levels in a microscope and uses the higher resolution representations for the regions requiring more detailed information for a classification decision. To achieve this, images were decomposed into multiresolution representations using the Gaussian pyramid approach [95]. This is followed by color space conversion and feature construction followed by feature extraction and feature selection at each resolution level. Once the classifier is confident enough at a particular resolution level, the system assigns a classification label (e.g., stroma-rich, stroma-poor or undifferentiated, poorly differentiating, differentiating) to the image tile. The resulting classification map from all image tiles forms the final classification map. The classification of a wholeslide image is achieved by dividing into smaller image tiles and processing each image tile independently in parallel on a cluster of computer nodes.

As an example, refer to Fig. 11, showing a hierarchical cascaded scheme for detecting suspicious areas on digitized prostate histopathology slides as presented in [96].

Fig. 12 shows the results of a hierarchical classifier for detection of prostate cancer from digitized histopathology. Fig. 12(a)

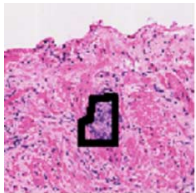

(a)

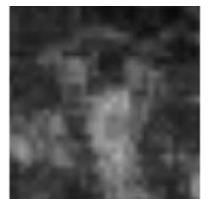

(b)

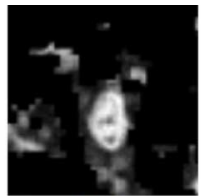

(c)

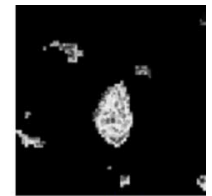

(d)
Fig. 12. Results from the hierarchical machine learning classifier. (a) Original image with the tumor region (ground truth) in black contour, (b) results at scale 1 , (c) results at scale 2 , and (d) results at scale 3 . Note that only areas determined as suspicious at lower scales are considered for further analysis at higher scales.

shows the original image with tumor outlined in black. The next three columns show the classifier results at increasing analysis scales. Pixels classified as "nontumor" at a lower magnification (scale) are discarded at the subsequent higher scale, reducing the number of pixels needed for analysis at higher scales. Additionally, the presence of more discriminating information at higher scales allows the classifier to better distinguish between tumor and nontumor pixels.

At lower resolutions of histological imagery, textural analysis is commonly used to capture tissue architecture, i.e., the overall pattern of glands, stroma and organ organization. For each digitized histological image several hundred corresponding feature scenes can be generated. Texture feature values are assigned to every pixel in the corresponding image. 3-D statistical, gradient, and Gabor filters can be extracted in order to analyze the scale, orientation, and anisotropic information of the region of interest. Filter operators are applied in order to extract features within local neighborhoods centered at every spatial location. At medium resolution, architectural arrangement of nuclei within each cancer grade can be described via several graph-based algorithms. At higher resolutions, nuclei and the margin and boundary appearance of ductal and glandular structures have proved to be of discriminatory importance. Many of these features are summarized in Tables I and II.

\section{Feature Selection, Dimensionality Reduction, and Manifold Learning}

1) Feature Selection: While humans have innate abilities to process and understand imagery, they do not tend to excel at 
explaining how they reach their decisions. As such, large feature sets are generated in the hopes that some subset of features incorporates the information used by the human expert for analysis. Therefore, many of the generated features could be redundant or irrelevant. Actually, a large set of features may possibly be detrimental to the classification performance, a phenomenon known as "the curse of dimensionality." Feature selection is a means to select the relevant and important features from a large set of features. This is an increasingly important area of research now that automated quantitative image analysis techniques are becoming more mainstream.

Feature selection in histopathological image analysis provides several benefits in addition to improving accuracy. Since images tend to be relatively large, a smaller subset of features needs to be calculated, reducing the computational complexity of classification algorithms. In some applications, it may be preferable to sacrifice the overall performance slightly if this sacrifice greatly reduces the number of selected features. A smaller number of features would also make it easier to explain the underlying model and improve the chances of generalization of the developed system. Additionally, in a multiresolution framework, a set of features proven useful at a given resolution may not be relevant at another resolution, even within the same image. A feature selection algorithm helps determine which features should be used at a given resolution.

An optimal feature selection method would require an exhaustive search, which is not practical for a large set of features generated from a large dataset. Therefore, several heuristic algorithms have been developed, which use classification accuracy as the optimality criterion. Well-known feature selection methods include the sequential search methods, namely sequential forward selection (SFS) and sequential backward selection (SBS) [97]. SFS works by sequentially adding the feature that most improves the classification performance; similarly, SBS begins with the entire feature set and sequentially removes the feature that most improves the classification performance. Both SFS and SBS suffer from the "nesting effect" whereby features that are selected (SFS) or discarded (SBS) cannot be revisited in a later step and are thus sub-optimal [97]. The use of floating search methods, sequential floating forward search (SFFS) and sequential floating backward search (SFBS), in which previously selected or discarded features can be re-evaluated at later steps avoids the nesting problem [97]. While these methods still cannot guarantee optimality of the selected feature subset, they have been shown to perform very well compared to other feature selection methods [98] and are, furthermore, much more computationally efficient [97]. SFFS is one of the most commonly encountered feature selection methods in pathology image analysis literature.

More recent feature selection research has focused on such methods as genetic algorithms, simulated annealing, boosting [99] and grafting [100]. A taxonomy of feature selection algorithms is presented in [98]. Genetic algorithms and simulated annealing are applications of traditional optimization techniques to feature selection. Boosting, which will be explained in Section VI-C, basically acts as a greedy feature selection process. Grafting (from "gradient feature testing") [100] is based on an elegant formulation of the feature selection problem, whereby the classification of the underlying data and the feature selection process are not separated. Within the grafting framework, a loss function is used that shows preference for classifiers that separate the data with larger margins. Grafting also provides an efficient framework for selection of relevant features. Feature selection based on a measure of discriminatory power was proposed in [101], whereby the authors compute the discriminatory power of each of the wavelet packet sub-bands (features) using a dissimilarity measure between approximated probability density functions for different classes. Derived features are then sorted according to the discriminatory power values associated with the corresponding features.

2) Dimensionality Reduction: While feature selection aims to select features (and reduce the feature dimensionality) that best optimize some criterion related to the class labels of the data (e.g., classification performance), dimensionality reduction techniques aim to reduce dimensionality based on some other criterion. Three well-known and commonly used methods of linear dimensionality reduction are principal component analysis (PCA), independent component analysis (ICA), and linear discriminant analysis (LDA).

Principal component analysis (PCA) [102] looks to find a new orthogonal coordinate system whereby the maximum variance of the data is incorporated in the first few dimensions. Projection of the data onto the individual coordinates encompasses varying degrees of variance; the first coordinate encompasses the largest variance in the data, the second coordinate the next largest variance, and so forth.

On the other hand, the LDA is a supervised method; it thus requires class labels for each data sample, mapping the data onto a lower dimensional sub-space that best discriminates data. The goal is to find the mapping, where the sum of distances between samples in different classes is maximized; while the sum of distances between samples in same classes is minimized. LDA can also be formulated in terms of eigenanalysis. A comprehensive discussion of PCA and LDA can be found in [103].

Independent component analysis [104], looks to find some mixing matrix such that a mixture of the observations (features) are statistically independent. This provides a stronger constraint on the resulting components than PCA, which only requires that the components be uncorrelated. This is why it is particularly well suited for decorrelating independent components from hyperspectral data. Rajpoot and Rajpoot have shown ICA to perform well for extracting three independent components corresponding to three tissue types for segmentation of hyperspectral images of colon histology samples. ICA, however, provides no ranking of the resulting independent components, as does PCA. There are a variety of methods for calculating the independent components (refer to [104]), which are generally very computationally intensive. ICA is a higher order method that seeks linear projections, not necessarily orthogonal to each other, as in the case of PCA.

3) Manifold Learning: Recently, nonlinear dimensionality reduction methods have become popular in learning applications. These methods overcome a major limitation of linear dimensionality reduction methods, which assume that geometrical structure of the high-dimensional feature space is linear. In reality, high-dimensional feature spaces comprise of highly 


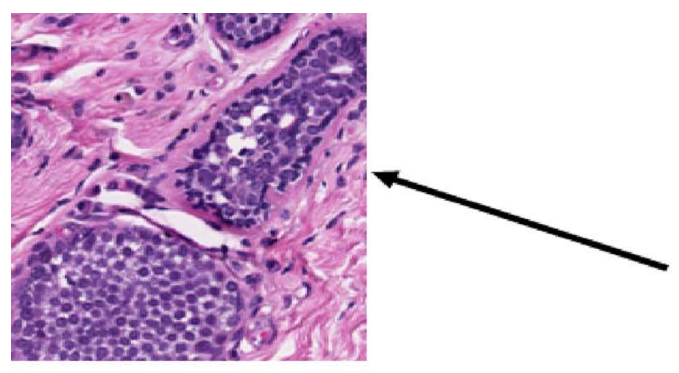

(a)

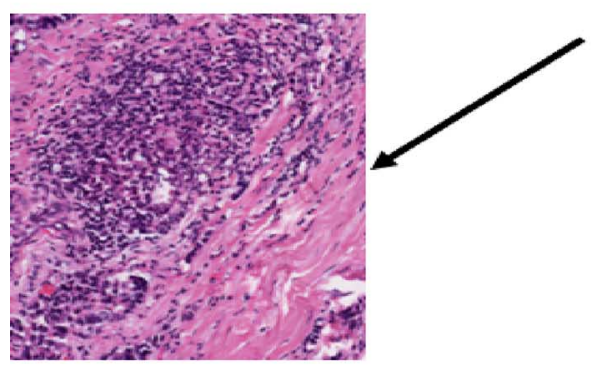

(b)

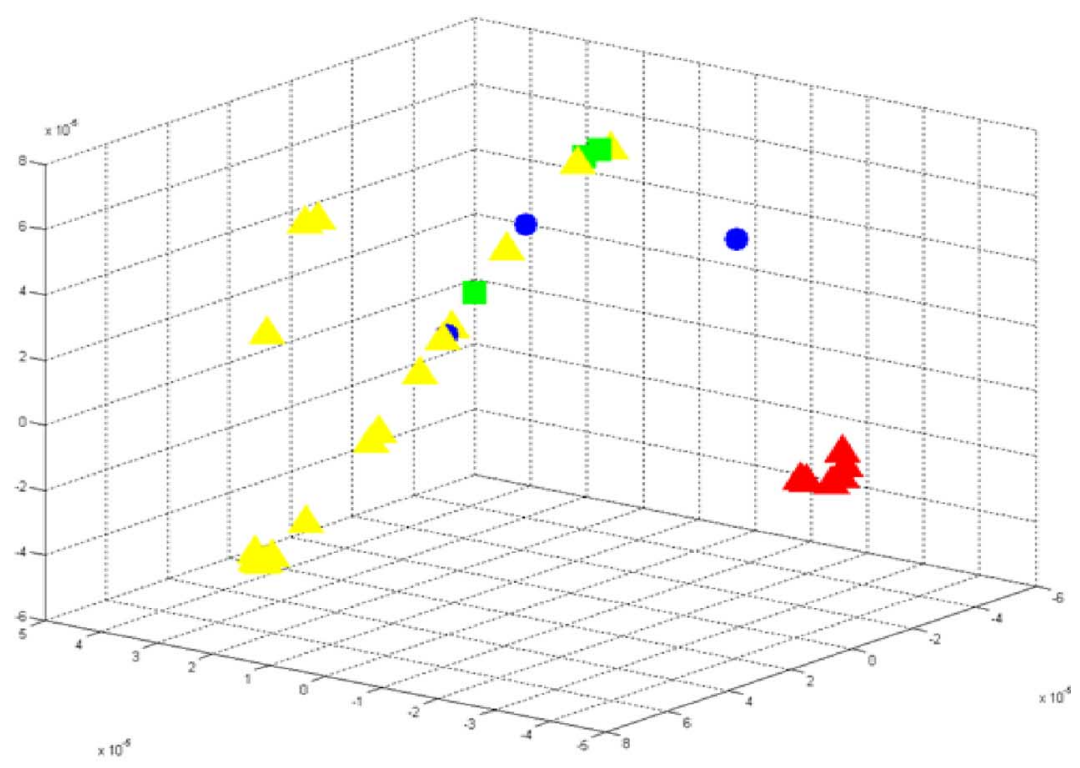

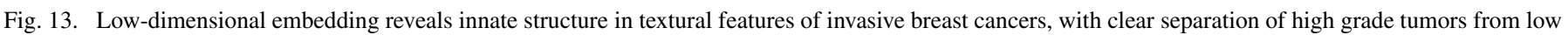

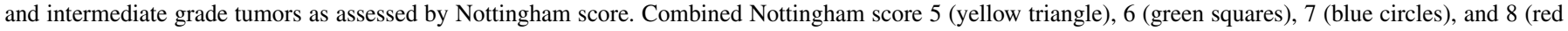
triangles). The score of 8 corresponds to high grade tumors. (a) Low grade (Yellow triangles). (b) High grade (Red triangles).

nonlinear structures and locality preserving dimensionality reduction methods are highly sought after. Manifold learning is a method of reducing a data set from $M$ to $N$ dimensions, where $N<M$ while preserving inter- and intra-class relationships between the data. This is done by projecting the data into a low-dimensional feature space in such a way to preserve high dimensional object adjacency. Many manifold learning algorithms have been constructed over the years to deal with different types of data.

Graph embedding constructs a confusion matrix $Y$ describing the similarity between any two images $\mathfrak{C}_{p}$ and $\mathfrak{C}_{\mathrm{q}}$ with feature vectors $\boldsymbol{F}_{p}$ and $\boldsymbol{F}_{\mathrm{q}}$, respectively, where $p, q \in\{1,2, \ldots, k\}$ and $k$ is the total number of images in the data set

$$
Y(p, q)=e^{-\left\|\mathrm{F}_{p}-\mathrm{F}_{\mathrm{q}}\right\|} \in \mathfrak{R}^{k \times k}
$$

The embedding vector $X$ is obtained from the maximization of the function

$$
\mathfrak{E}_{Y}(X)=\frac{2 \eta \mathrm{X}^{\mathrm{T}}(\mathrm{D}-\mathrm{Y}) \mathrm{X}}{\mathrm{X}^{\mathrm{T}} \mathrm{DX}}
$$

where $\mathrm{D}$ is the so-called degree matrix, with nonzero values being along the diagonal $D(p, p)=\sum_{\mathrm{q}} Y(p, q)$ and $\eta=k-1$. The $k$ dimensional embedding space is defined by the eigenvectors corresponding to the smallest $N$ eigenvalues of $(D-$ $Y) X=\lambda D X$. The value of $N$ is generally optimized by obtaining classification accuracies for $N \in\{1,2, \ldots, 10\}$ and selecting the $N$ that provided the highest accuracy for each classification task. For image $\mathfrak{C}$, the feature vector $\boldsymbol{F}$ given as input to the graph embedding algorithm produces an $N$-dimensional eigenvector $X(\mathfrak{C})=\left[e_{j}(\mathfrak{C}) \mid j \in\{1,2, \ldots \ldots, N\}\right]$, where $e_{j}(\mathfrak{C})$ is the principal eigenvalue associated with $\mathfrak{E}$.
In [92], a graph embedding algorithm employing the normalized cuts algorithm was used to reconstruct the underlying manifold on which different breast cancer grades were distributed. Fig. 13 shows the embedding of different grades of breast cancer histopathology (low, intermediate, high) on the reconstructed manifold; low grades (yellow triangles), intermediate grades (green squares and blue circles), and high grades (red triangles). The manifold captures the biological transformation of the disease in its transition from low to high-grade cancer.

Manifold learning has also been shown to be useful for shapebased classification of prostate nuclei [105]. Rajpoot et al. [105] employ diffusion maps [106] in order to reduce the dimensionality of shape descriptors down to two dimensions and a fast classification algorithm is derived based on a simple thresholding of the diffusion coordinates.

\section{Classification And Sub-CEllular Quantification}

For histopathology imagery, unlike some other applications of image analysis, one of the primary considerations in the choice of a classifier is its ability to deal with large, highly dense datasets. Also due to multiple image scales at which relevant information may be extracted from histological imagery, use of an ensemble of classifiers as opposed to a single classifier has been proposed.

\section{A. Multiclassifier Ensemble Schemes}

Theoretical and empirical results alike have established that, in terms of accuracy, ensembles of classifiers generally outperform monolithic solutions. Learning ensembles or multiple classifier systems are methods for improving classification accuracy through aggregation of several similar classifiers' predictions 
TABLE III

The Average Accuracy Results of the Diseased-Healthy-Damaged Classification on the Test Set by Using Five Different Approaches.

\begin{tabular}{|l|l|l|l|l|l|}
\hline Brain Tissue & Cell Graph & Cell Dist & Textural & Intensity & Voronoi \\
\hline Overall & $95.45( \pm) 1.33$ & $84.34( \pm) 5.52$ & $89.03( \pm) 1.51$ & $62.50( \pm) 3.76$ & $93.35( \pm) 0.47$ \\
\hline Diseased & $95.14( \pm) 2.03$ & $80.66( \pm) 7.96$ & $89.04( \pm) 2.01$ & $62.27( \pm) 3.94$ & $92.73( \pm) 0.27$ \\
\hline Healthy & $98.15( \pm) 0.00$ & $98.15( \pm) 0.00$ & $97.22( \pm) 1.44$ & $91.60( \pm) 6.24$ & $98.15( \pm) 0.00$ \\
\hline Damaged & $92.50( \pm) 1.76$ & $64.38( \pm) 14.87$ & $75.21( \pm) 4.56$ & $14.58( \pm) 4.21$ & $88.44( \pm) 3.09$ \\
\hline
\end{tabular}

and thereby reducing either the bias or variance of the individual classifiers [107].

1) A Support Vector Machines (SVM): SVMs project a set of training data $E$ representing two different classes into a highdimensional space by means of a kernel function $\mathbf{K}$. The algorithm then generates a discriminating hyper-plane to separate out the classes in such a way to maximize a cost function. Testing data is then projected into the high-dimensional space via $\mathbf{K}$, and the test data is classified based on where it falls with respect to the hyper-plane. The kernel function $\mathbf{K}(\cdot, \cdot)$ defines the method in which data is projected into the high-dimensional space. A commonly used kernel known as the radial basis function has been employed to distinguish between three different prostate tissue classes [108]. Radial basis functions with a grid search for their parameters have also been used to differentiate colon adenocarcinoma histopathology images from benign histopathology images [109] and to classify four different subtypes of meningioma [101].

2) Adaboost: The AdaBoost is an adaptive algorithm in the sense it combines a number of weak classifiers to generate a strong classifier. Image pixels determined as diseased by a pathologist during the training stage are used to generate probability density functions (pdfs) for each of the individual texture features $\Phi_{j}$, for $j \in\{1, \ldots, K\}$ which are considered as weak classifiers [110]. Bayes Theorem is then used to generate likelihood scenes $L_{j}=\left(C_{j}, l j \in\{1, \ldots, K\}\right)$ for each $\Phi_{j}$ which constitute the weak learners. These are combined by the AdaBoost algorithm into a strong classifier $\Pi^{j}=\sum_{i=1}^{\mathrm{T}} \alpha_{i}^{j} l_{i}^{j}$ where for every pixel $c^{j} \in C^{j}, \Pi^{j}\left(c^{j}\right)$ is the combined likelihood that pixel $c^{j}$ belongs to cancer class $\omega_{T}$, $\alpha_{i}^{j}$ is the weight determined during training for feature $\Phi_{i}$, and $\mathrm{T}$ is the number of iterations.

In [110] a hierarchical boosted cascade scheme for detecting suspicious areas on digitized prostate histopathology, inspired by the work of Viola and Jones [111] was presented. Efficient and accurate analysis is performed by first only detecting those areas that are found to be suspicious at lower scales. Analysis at subsequent higher magnifications is limited to those regions deemed to be suspicious at lower scales. Pixels classified as "nontumor" at a lower magnification (scale) are discarded at the subsequent higher scale, reducing the number of pixels needed for analysis at higher scales. The process is repeated using an increasingly larger number of image features and an increasing classification threshold at each iteration. Qualitative results with a hierarchical Boosted classifier at three different image resolutions are shown in Fig. 14.

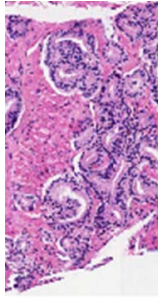

(a)

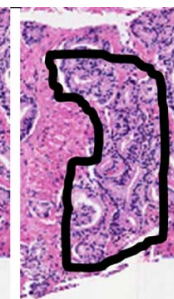

(b)

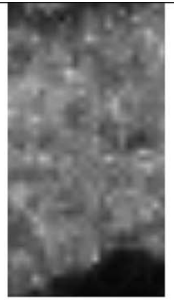

(c)

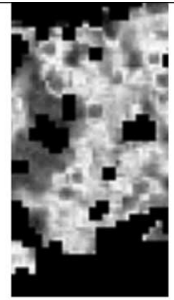

(d)

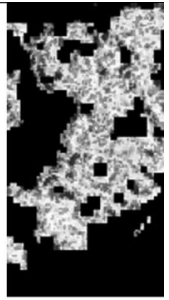

(e)
Fig. 14. From left to right, (a) A digitized histopathology image, (b) cancer extent delineated in black by an expert pathologist, and cancer probability images generated by an Adaboost classifier at (c) low-, (d) intermediate, and (e) high image resolutions.

\section{B. Disease Discrimination Based on Graph-Based Features}

Table III shows the average accuracy and corresponding standard deviation over 20 runs of randomized cross validation based classification of the diseased-healthy-damaged studies by using five different approaches: 1) the cell graph approach; 2) the cell-distribution approach in which features are extracted from the spatial distribution of cells without considering their pair-wise spatial relations (i.e., without considering the edge information); 3) the textural approach in which features are derived from the gray-level interdependency of pixels in a tissue image; 4) the intensity-based approach in which features are derived from the intensity values of pixels in a tissue image; and 5) the Voronoi diagram-based approach in which features are extracted by making use of the spatial interrelationships of adjacent cells [112], [113].

\section{Grade-Based Classification of Histopathology Imagery}

The classification of histopathology imagery is often the ultimate goal in image analysis, particularly in cancer applications. Features derived from segmented nuclei and glands from histopathology are usually a prerequisite to extracting higher level information regarding the state of the disease. For instance, the grading of prostate cancer by Jafari-Khouzani and Soltanian-Zadeh [114] yielded 97\% accuracy for H\&E stained imagery based on features derived from nuclear structures in histopathology. Weyn et al. [115] reported $87.1 \%-96.8 \%$ accuracy in the correct diagnosis ( 3 diagnoses) of Feulgen-stained lung cancer specimens, $79.5 \%-92.3 \%$ accuracy in typing (3 types) malignant mesothelioma, and $74.3 \%-82.9 \%$ accuracy in the prognosis (3 classes of survival time) of malignant mesothelioma cases. Analysis of Feulgen-stained breast tissue sections by van de Wouwer et al. [116] found $67.1 \%$ accuracy 


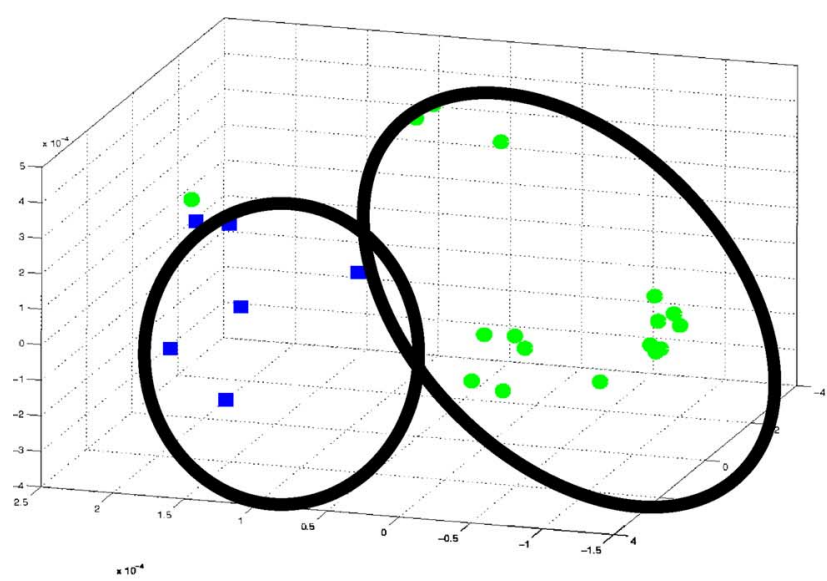

(a)

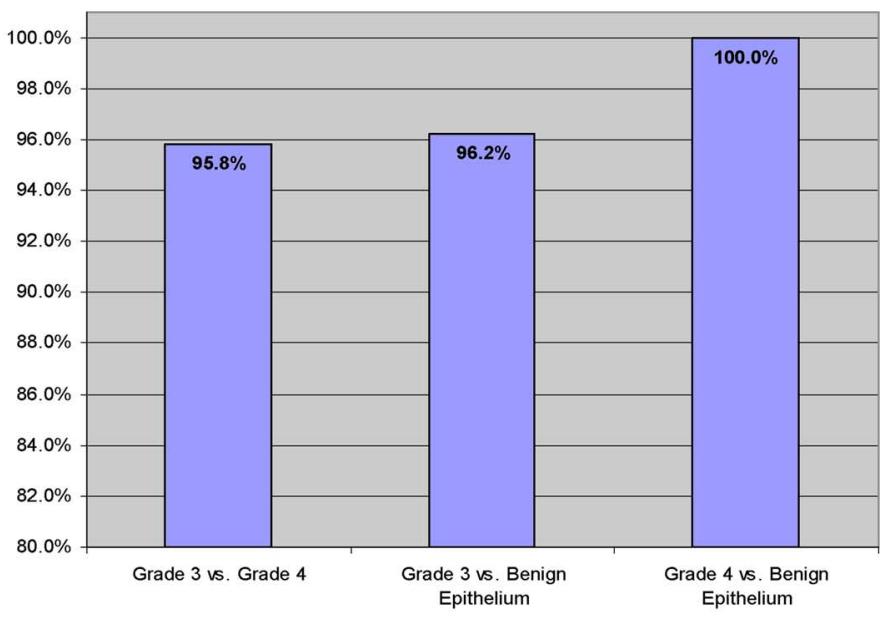

(b)

Fig. 15. (a) (left panel) Low dimensional representation (via nonlinear dimensionality reduction) of prostate cancer histopathology images (green circles are Grade 3 images and blue squares represent Grade 4 prostate cancer images). A nonlinear SVM is used to classify objects in the reduced dimensional space. (b) Right panel shows a classification accuracy of over $90 \%$ in distinguishing between Grade 3, Grade 4 images and comparable accuracy in distinguishing between benign stromal, epithelial, and prostate cancer tissue.

in clasifying nuclei as benign or malignant, but $100 \%$ classification on a patient level. Tabesh et al. [117] found $96.7 \%$ accuracy in discriminating between prostate tissue slides with cancer and no cancer, and $81 \%$ accuracy in the discrimination between low and high Gleason grades in the same imagery. Immunohistochemically stained colon mucosa allowed for an accuracy of $92 \%$ in classification of benign and malignant images by Esgiar et al. [118].

The classification of histopathology imagery using spatial architecture information as presented in Weyn et al. [115] resulted in $88.7 \%-96.8 \%$ accuracy in the diagnosis of lung cancer, $94.9 \%$ accuracy in the typing of malignant mesothelioma, and $80.0 \%-82.9 \%$ accuracy in the prognosis of malignant mesothelioma for Feulgen-stained lung sections. The analysis of H\&E stained brain tissue by Demir et al. [112] gave 95.5\%-97.1\% accuracy in the discrimination between benign and cancerous tissue. Keenan et al. [119] reported accuracies of $62.3 \%-76.5 \%$ in the grading of $\mathrm{H} \& \mathrm{E}$ stained cervical tissue.

Fig. 15(a) shows the low dimensional embedding of the high dimensional attribute space via locally linear embedding of 20 images representing prostate cancer grades 3 (green circles) and 4 (blue squares). Each image is displayed as a point in 3D eigenspace. The clustering clearly shows very good discrimination between these 2 classes which clinically is the most challenging problem in terms of Gleason grading. Fig. 15(b) shows bar plots reflecting the classification accuracy obtained via a supervised classifier in distinguishing between pairs of tissue classes - grade 314 , grade 3 vs. benign epithelium, and grade 4 vs. benign epithelium via a SVM classifier. Note that in every case the classification accuracy is over $90 \%$.

\section{Sub-Cellular Quantification}

Quantifying expression levels of proteins with sub-cellular resolution is critical to many applications ranging from biomarker discovery, pharmaceutical research, and systems biology to treatment planning. In this section, a fully automated method to quantify the expression levels of target proteins in immunofluorescently stained samples in tissue micro arrays (TMAs) is presented. Kolmogorov-Smirnov (KS) statistics, a well-known method in statistics to test if two distributions are different from each other, can be used to compute the relative expression levels in each of the epithelial and nonepithelial tissue regions. After the sub-cellular compartments are determined using membrane and nuclear markers, the distribution of target proteins in each of these compartments are calculated. The estimated distribution comprises target distribution from the expressed regions as well as nonspecific background binding and tissue autofluorescence that may have left out after the AF removal step. A signed KS distance (sKS) can be defined as the product of the standard KS distance and a sign function $( \pm 1)$ to indicate which compartment is expressed. For example if sign function is computed between the nuclear and the membrane expression levels, a negative sKS distance will indicate a nuclear expression, and a positive sign will indicate membrane expression. While the magnitude of the sKS distance indicates how strong the expression level difference is between these compartments, its sign indicates in which compartment the protein is expressed.

The standard KS distance is defined as the maximum separation between the cumulative distribution functions (CDF) of two data sets. The CDF of a target protein in any compartment can be calculated by integrating its intensity distribution estimated using a Parzen window approach with Gaussian kernels. Fig. 16(c) shows the CDF of the target distributions shown in Fig. 16(a) (green color), on each of the segmented sub-cellular regions; nuclei (blue), membrane (red), and cytoplasm (green) shown in Fig. 16(b). The CDFs clearly indicate the overexpression of the nuclear region (blue plot) where approximately $10 \%$ of the nuclear pixels express intensity values more than 50 , as opposed to a very small percentage for other compartments. The sign of the sKS is determined based on sign of the difference between the CDF of the first compartment and the CDF of the 


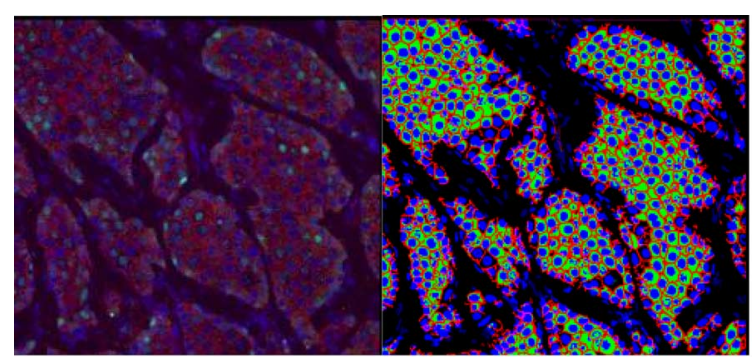

KS distance of the ER expression on the Epithelial Compartments

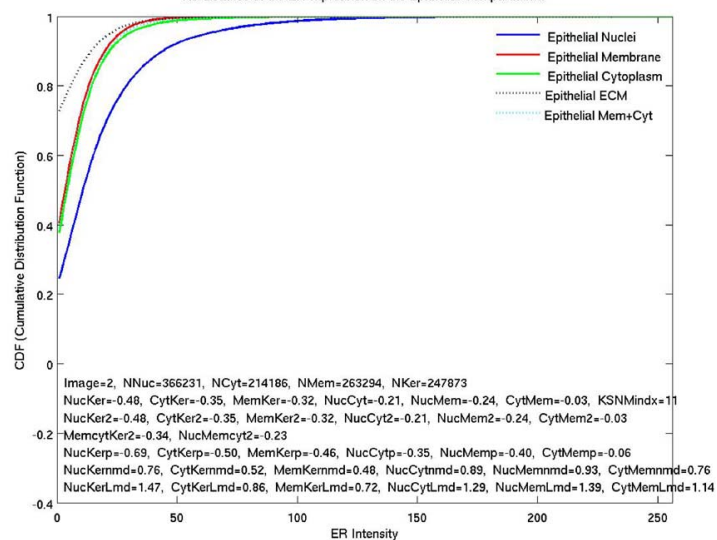

(a)

(b)

(c)

Fig. 16. (a) A histological section stained with nuclear (DAPI-Blue), membrane (Pan-cadherin, red), and a target protein (Estrogen Receptor (ER), green). (b) Automatically segmented sub-cellular regions; membrane (red), nuclei (blue), cytoplasm (green). Dark colors show the nonepithelial regions. (c) CDF of the ER distributions (nuclei in blue, membrane in red and cytoplasm in green plots).

second compartment at the protein expression level with highest CDF separation.

The sKS metric on 123 TMA images from 55 patients (some patients are represented as multiple tissue cores) stained with DAPI (nuclei), pan-cadherin (membrane), keratin (tumor/epithelial mask), and ER markers were tested. DAPI and pan-cadherin are used to segment the sub-cellular compartments, and keratin to segment the epithelial mask. Then the distribution of the ER protein is calculated on each of the epithelial sub-cellular regions. ER is expected to partially or fully express in the nuclear region for ER positive patients. A target distribution is usually observed mixed with nonspecific expression and auto-fluorescence (AF). The sKS based metric was compared to the majority decision of a group of 19 observers scoring estrogen receptor (ER) status, and achieved $96 \%$ detection rate with $90 \%$ specificity. The presented methods have applications from diagnostics to biomarker discovery and pharmaceutical research.

\section{LoOKIng AheAd: Future TRENDS, Open PROBlems}

Since histopathological image analysis is inherently a crossdisciplinary area, there are unique challenges to the dissemination of research results. One of these is the wide range of publications in which research is published. While there are a few journals that focus on the automated analysis of medical imagery, the majority of histopathological image analysis tends to be published in the leading journals of the researchers' field (pathology, computer vision, etc.). Additionally, there is a need for more evidence regarding the clinical applicability and importance of automated histopathology image analysis methods. We have mentioned areas throughout this paper for which we anticipate ongoing research to have a clear and tangible affect on clinical and pathology workflow.

Comparison of the various methods presented in the literature is difficult, since each research team uses their own dataset and presents their results with different metrics. There is a great need for standard datasets and ground truth for validation of methods. As an example, researchers at the University of South Florida have put together a database of digital mammograms. ${ }^{1}$ While the variety of conditions studied in histopathology image analysis is greater, it is still important that standard datasets be compiled as well as a standard metric of performance. This will allow for direct comparison of the variety of analysis methods being reported in the literature. An additional complication is the variety of analyses performed on the histopathology imagery. Thus, there is a need for a dataset with ground truth pertaining to all the analyses described in this paper.

Going forward, clinical annotation of histopathology data will be a large bottleneck in the evaluation of histopathology related CAD algorithms. Apart from the time constraints on the pathologist to generate this data, the process should be streamlined with active communication between the image analysis scientists and the clinicians with regard to the sort of annotation required, the format and scale at which the annotation is generated, and the ease with which the data can be shared (since histopathology files typically tend to be very large). For instance, the sophistication of annotation required to train a CAD system to distinguish cancerous versus noncancerous regions on pathology images may be very different than the annotation detail required to train a classifier to distinguish grades of cancer. While for the former problem the annotation could be done on a coarser scale (lower resolution), the latter annotation may require explicit segmentation of glands and nuclei, a far more laborious and time consuming process. Due to the large size of pathological images, usually it is not possible to process the whole image on a single-core processor. Therefore, the whole image may be divided into tiles and each tile is processed independently. As a consequence, automatic load balancing in the distribution of the cases to different processors need to be handled carefully [120]. Additionally, the processing can be accelerated even further by the use of graphical processing units (GPUs), cell blades, or any other emerging high-performance architecture [121].

Histopathological image analysis system evaluation needs to be carried out in a statistical framework. Depending on whether it is a problem of detection (e.g., nuclei detection) or characterization (e.g., grading), some commonly accepted evaluation methodologies need to be followed. Some of these methods, e.g., receiver operating characteristics (ROC) and free response operating characteristics (FROC), have been successfully used for many years in radiology [122]. These techniques could be adopted or adapted accordingly. The level and detailed of quantitative evaluation will vary as a function of the specific problem being addressed. For instance, in order to evaluate a nuclear segmentation algorithm on a digitized histological section containing several tens of thousands of nuclei, it is unreasonable to

\footnotetext{
${ }^{1} \mathrm{http}: / /$ marathon.csee.usf.edu/Mammography/Database.html
} 


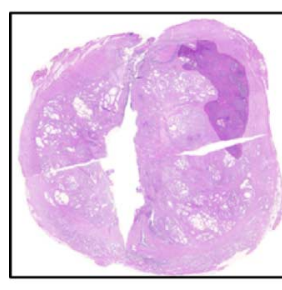

(a)

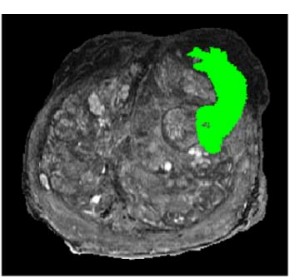

(b)

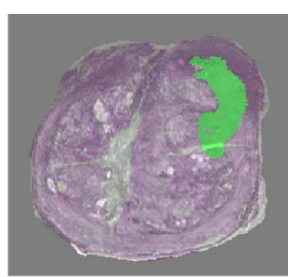

(c)
Fig. 17. (a) Histology section of prostate gland with $\mathrm{CaP}$ extent stained in purple (upper right) and corresponding mapping of $\mathrm{CaP}$ extent via COFEMI onto (b) MRI (CaP extent shown in green). (c) Overlay of histological and MRI prostate sections following registration.

expect that a human reader will be able to manually annotate all nuclei. Evaluation of the scheme may have to be performed on randomly chosen segments of the image. Similarly, if the ultimate objective of the CAD algorithm is, for instance, cancer grading, perfect segmentation of histological structures may not guarantee perfect grade-based classification. Evaluation should hence be tailored towards the ultimate objective that the CAD algorithm is being employed for. Additionally, special attention needs to be paid to clearly separate training and testing datasets and explain the evaluation methodology.

\section{A. Multimodal Data Fusion/Registration}

While digital pathology offers very interesting, highly dense data, one of the exciting challenges will be in the area of multimodal data fusion. One of the big open questions, especially as it pertains to personalized medicine, will be the use of multimodal data classifiers to be able to make therapy recommendations. This will require solving questions both in terms of data alignment and in terms of knowledge representation for fusion of heterogeneous sources of data, in order to answer questions that go beyond just diagnosis, such as theragnosis (therapy prediction) and prognosis.

$\mathrm{H} \& \mathrm{E}$ staining is traditionally used for histopathology imaging. Several other modalities exist for imaging of the tissue, each offering its own advantages and limitations. Combining images from different modalities, therefore, may seem to be an attractive proposition, although it does not come without its own challenges, most importantly registration, not to mention the extra cost associated with imaging, storage, and computational time. Registration of image data across the different modalities and fusion of the information contained therein result in a powerful resource of information for diagnosis and prognosis purposes. Fusion methods have been developed for images from different microscopy imaging methods [26] and micro-scale histopathology and large-scale MR images [123]-[125].

Madabhushi et al. [126] have been developing computerized detection methods for prostate cancer from high-resolution multimodal MRI . A prerequisite to training a supervised classifier to identify prostate cancer $(\mathrm{CaP})$ on $\mathrm{MRI}$ is the ability to precisely determine spatial extent of $\mathrm{CaP}$ on the radiological imaging modality. CaP can be precisely determined on whole mount histopathology specimens [Fig. 17(a)] which can then be mapped onto MRI [Fig. 17(b)]. Fig. 17(c) shows the result of registering [Fig. 17(b)] the 2-D MRI slice to the histological section [Fig. 17(a)]. This requires the use of sophisticated and robust multimodal deformable registration methods to account for (a) deformations and tissue loss in the whole mount histological specimens during acquisition, and (b) ability to overcome intensity and feature differences between the two modalities (histopathology and MRI). In [123], [124] a rigid registration scheme called combined feature ensemble based mutual information (COFEMI) was presented that used alternate feature representations of the target and source images to be registered to facilitate the alignment.

\section{B. Correlating Histological Signatures With Protein and Gene Expression}

Multiplexing, imaging of a tissue sample with several antibodies simultaneously, allows correlation of characteristic patterns in histopathology images to expression of proteins. Teverovskiy et al. [127] recently proposed a novel scheme for automated localization and quantification of the expression of protein biomarkers using a DAPI counter-stain and three other biomarkers. They showed it to be useful for predicting recurrence of prostate cancer in patients undergoing prostatectomy. Recently, it has become clear that information regarding expression of certain proteins related to the onset of cancer is not sufficient. Analyzing multiple-stained histopathology images can help identify oncogenesis-induced changes in sub-cellular location patterns of proteins. Glory et al. [128] proposed a novel approach to compare the sub-cellular location of proteins between normal and cancerous tissues. Such a method can also be used for identification of proteins to be used as potential biomarkers.

\section{Exploratory Histopathology Image Analysis}

Exploratory analysis of histopathology images can help in finding salient diagnostic features used by humans, associating them with the computed features, and visualizing relationships between different features in high-dimensional spaces. Lessmann et al. [129] have proposed the use of self-organizing maps (SOMs) for exploratory analysis of their wavelet-based feature space. The SOM-based visualization of the feature space allowed the authors of [129] to establish a correlation between single features and histologically relevant image structures, making the selection of a subset of clinically important features possible. Iglesias-Rozas and Hopf [130] showed that SOMs can be effectively employed to correctly classify different subtypes of human Glioblastomas (GB) and also to select significant histological and clinical or genetic variables. Alternatively, dimensionality reduction methods may offer a way of looking at trends and patterns in the data in a reduced dimensional space [131]-[133].

\section{Computer-Aided Prognosis}

The use of computer-aided diagnosis for digitized histopathology could begin to be employed for disease prognostics, allowing physicians to predict which patients may be susceptible to a particular disease and also predicting disease outcome and survival. For instance, since grade is known to be correlated to outcome (high grade correlates to worse outcome), image-based predictors could be used to predict disease recurrence and survival based on analysis of biopsy specimens alone. This would have significant translational implications in 
that; more expensive molecular assays may not be required for predicting disease.

While there may be a small minority of researchers who are experts in both computer vision and pathology, the vast majority of histopathology image analysis researchers are computer vision researchers. As such, it is important to maintain a constant collaboration with clinical and research pathologists throughout the research process. There are unique challenges to analysis of medical imagery, particularly in the performances required for eventual use of the technique in a clinical setting. It is the pathologist who can best provide the feedback on the performance of the system, as well as suggesting new avenues of research that would provide beneficial information to the pathologist community. Additionally, it is the pathologist that is best equipped to interpret the analysis results in light of underlying biological mechanisms which, in turn, may lead to new research ideas. Similarly, where appropriate it might be pertinent to include the oncologist and radiologist within the algorithmic development and evaluation loop as well.

\section{ACKNOWLEDGMENT}

M. N. Gurcan would like to thank O. Sertel and K. Boussaid for carefully reviewing the manuscript and for useful discussions.

\section{REFERENCES}

[1] A. J. Mendez, P. G. Tahoces, M. J. Lado, M. Souto, and J. J. Vidal, "Computer-aided diagnosis: Automatic detection of malignant masses in digitized mammograms," Med Phys., vol. 25, pp. 957-64, Jun. 1998

[2] J. Tang, R. Rangayyan, J. Xu, I. E. Naqa, and Y. Yang, "Computeraided detection and diagnosis of breast cancer with mammography: Recent advances," IEEE Trans. Inf. Technol. Biomed., vol. 13, no. 2, pp. 236-251, Mar. 2009.

[3] R. Rubin, D. Strayer, E. Rubin, and J. McDonald, Rubin's Pathology: Clinicopathologic Foundations of Medicine. Baltimore, MD: Lippincott Williams \& Wilkins, 2007.

[4] K. L. Weind, C. F. Maier, B. K. Rutt, and M. Moussa, "Invasive carcinomas and fibroadenomas of the breast: Comparison of microvessel distributions-implications for imaging modalities," Radiology, vol. 208, pp. 477-83, Aug. 1998.

[5] P. H. Bartels, D. Thompson, M. Bibbo, and J. E. Weber, "Bayesian belief networks in quantitative histopathology," Anal. Quant. Cytol. Histol., vol. 14, pp. 459-73, Dec. 1992.

[6] P. W. Hamilton, N. Anderson, P. H. Bartels, and D. Thompson, "Expert system support using Bayesian belief networks in the diagnosis of fine needle aspiration biopsy specimens of the breast," J Clin. Pathol., vol. 47, pp. 329-36, Apr. 1994.

[7] W. C. Allsbrook Jr., K. A. Mangold, M. H. Johnson, R. B. Lane, C. G. Lane, and J. I. Epstein, "Interobserver reproducibility of Gleason grading of prostatic carcinoma: General pathologist," Hum. Pathol., vol. 32, pp. 81-8, Jan. 2001.

[8] C. R. King and J. P. Long, "Prostate biopsy grading errors: A sampling problem?," Int. J. Cancer, vol. 90, pp. 326-30, Dec. 2000.

[9] B. Djavan, K. Kadesky, B. Klopukh, M. Marberger, and C. G. Roehrborn, "Gleason scores from prostate biopsies obtained with 18 -gauge biopsy needles poorly predict Gleason scores of radical prostatectomy specimens," Eur. Urol.., vol. 33, pp. 261-70, 1998.

[10] E. Ruijter, G. van Leenders, G. Miller, F. Debruyne, and C. van de Kaa, "Errors in histological grading by prostatic needle biopsy specimens: Frequency and predisposing factors," J Pathol., vol. 192, pp. 229-33, Oct. 2000.

[11] L. Egevad, W. C. Allsbrook Jr., and J. I. Epstein, "Current practice of Gleason grading among genitourinary pathologists," Hum. Pathol., vol. 36, pp. 5-9, Jan. 2005.

[12] M. Ladekarl, "Objective malignancy grading: A review emphasizing unbiased stereology applied to breast tumors," APMIS Suppl., vol. 79, pp. 1-34, 1998.
[13] M. G. Daniel and J. Luthringer, "Gleason grade migration: changes in prostate cancer grade in the contemporary era," Departments of Pathology and Medicine, Cedars-Sinai, Los Angeles, CA, 2001, vol. 9.3, Reprinted from PCRI Insights.

[14] F. S. F. T. A. Stamey, J. E. McNeal, E. A. Redwine, A. S. Whittemore, and H.-P. Schmid, "Localized prostate cancer. Relationship of tumor volume to clinical significance for treatment of prostate cancer," Cancer, vol. 71, pp. 933-938, 1993.

[15] M. L. Giger, H. P. Chan, and J. Boone, "Anniversary paper: History and status of CAD and quantitative image analysis: The role of medical physics and AAPM," Med. Phys., vol. 35, pp. 5799-820, Dec. 2008

[16] T. M. Haygood, G. J. Whitman, E. N. Atkinson, R. G. Nikolova, S. Y. Sandoval, and P. J. Dempsey, "Results of a survey on digital screening mammography: Prevalence, efficiency, and use of ancillary diagnostic AIDS," J. Amer. Coll. Radiol., vol. 5, pp. 585-92, Apr. 2008.

[17] R. Schulz-Wendtland, K. P. Hermann, T. Wacker, and W. Bautz, "Current situation and future perspectives of digital mammography," Radiologe, vol. 48, pp. 324-34, Apr. 2008.

[18] A. Azari and S. Nikzad, "Computer-assisted implantology: Historical background and potential outcomes-A review," Int. J. Med. Robot., vol. 4, pp. 95-104, Jun. 2008.

[19] F. Sardanelli, G. M. Giuseppetti, G. Canavese, L. Cataliotti, S. Corcione, E. Cossu, M. Federico, L. Marotti, L. Martincich, P. Panizza, F. Podo, M. Rosselli Del Turco, C. Zuiani, C. Alfano, M. Bazzocchi, P. Belli, S. Bianchi, A. Cilotti, M. Calabrese, L. Carbonaro, L. Cortesi, C. Di Maggio, A. Del Maschio, A. Esseridou, A. Fausto, M. Gennaro, R. Girometti, R. Ienzi, A. Luini, S. Manoukian, S. Morassutt, D. Morrone, J. Nori, A. Orlacchio, F. Pane, P. Panzarola, R. Ponzone, G. Simonetti, P. Torricelli, and G. Valeri, "Indications for breast magnetic resonance imaging. Consensus document "Attualita in senologia", Florence 2007," Radiol. Med., vol. 113, pp. 1085-1095, Dec. 2008.

[20] K. Doi, "Computer-aided diagnosis in medical imaging: Historical review, current status and future potential," Comput. Med. Imag. Graph., vol. 31, pp. 198-211, Jun.-Jul. 2007.

[21] E. Sakka, A. Prentza, and D. Koutsouris, "Classification algorithms for microcalcifications in mammograms (Review)," Oncol. Rep., vol. 15, pp. 1049-55, 2006.

[22] L. R. Zhu, Y. Zhang, X. Y. Wang, and Q. P. Liao, "Assessment of magnetic resonance imaging in preoperative staging of endometrial carcinoma," Beijing Da Xиe Xue Bao, vol. 38, pp. 249-51, Jun. 2006.

[23] A. C. Roque and T. C. Andre, "Mammography and computerized decision systems: a review," Ann. NY Acad. Sci., vol. 980, pp. 83-94, Dec. 2002.

[24] H. Fox, "Is H\&E morphology coming to an end?," Brit. Med. J., vol. 53 , p. $38,2000$.

[25] R. Camp, G. Chung, and D. Rimm, "Automated subcellular localization and quantification of protein expression in tissue microarrays," Nature Med., vol. 8, pp. 1323-1328, 2002.

[26] A. Can, M. Bello, X. Tao, M. Gerdes, A. Sood, M. Montalto, and F. Ginty, "Techniques for cellular quantitation of cancer biomarkers," in Microscopic Image Analysis for Life Science Applications. Norwood, MA: Artech House, 2008.

[27] F. Ginty, S. Adak, A. Can, M. Gerdes, M. Larsen, H. Cline, R. Filkins, Z. Pang, Q. Li, and M. Montalto, "The relative distribution of membranous and cytoplasmic met is a prognostic indicator in stage I and II colon cancer," Clin. Cancer Res., vol. 14, p. 3814, 2008.

[28] J. Price, A. Goodacre, K. Hahn, L. Hodgson, E. Hunter, S. Krajewski, R. Murphy, A. Rabinovich, J. Reed, and S. Heynen, "Advances in molecular labeling, high throughput imaging and machine intelligence portend powerful functional cellular biochemistry tools," J. Cell. Biochem., vol. 87, pp. 194-210, 2002.

[29] A. Can, M. Bello, H. E. Cline, X. Tao, F. Ginty, A. Sood, M. Gerdes, and M. Montalto, "Multi-modal imaging of histological tissue sections," in 5th IEEE Int. Symp. Biomed. Imag.: From Nano to Macro, 2008, pp. 288-291.

[30] W. Schubert, B. Bonnekoh, A. Pommer, L. Philipsen, R. Bockelmann, Y. Malykh, H. Gollnick, M. Friedenberger, M. Bode, and A. Dress, "Analyzing proteome topology and function by automated multidimensional fluorescence microscopy," Nature Biotechnol., vol. 24, pp. 1270-1278, 2006.

[31] J. Newberg and R. Murphy, "A framework for the automated analysis of subcellular patterns in human protein atlas images," J. Proteome Res., vol. 7, pp. 2300-2308, 2008.

[32] A. Rabinovich, S. Agarwal, C. Laris, J. Price, and S. Belongie, "Unsupervised color decomposition of histologically stained tissue samples," Adv. Neural Inf. Process. Syst., vol. 16, pp. 667-674. 
[33] D. Fernandez, R. Bhargava, S. Hewitt, and I. Levin, "Infrared spectroscopic imaging for histopathologic recognition," Nature Biotechnol., vol. 23, pp. 469-474, 2005.

[34] E. Blout and M. Fields, "On the infrared spectra of nucleic acids and certain of their components," Science, vol. 107, p. 252, 1948.

[35] E. R. Blout and R. C. Mellors, "Infrared spectra of tissues," Science, vol. 110 , pp. 137-138, 1949.

[36] D. Woernley, "Infrared absorption curves for normal and neoplastic tissues and related biological substances," Cancer Res., vol. 12, p. 516, 1952.

[37] W. Hollingworth, L. Medina, R. Lenkinski, D. Shibata, B. Bernal, D. Zurakowski, B. Comstock, and J. Jarvik, "Interrater reliability in assessing quality of diagnostic accuracy studies using the QUADAS toola preliminary assessment," Acad. Radiol., vol. 13, pp. 803-810, 2006.

[38] F. Howe and K. Opstad, "1 H MR spectroscopy of brain tumours and masses," NMR Biomed., vol. 16, pp. 123-131, 2003.

[39] L. M. McIntosh, J. R. Mansfield, A. N. Crowson, H. H. Mantsch, and M. Jackson, "Analysis and interpretation of infrared microscopic maps: Visualization and classification of skin components by digital staining and multivariate analysis," Biospectroscopy, vol. 5, pp. 165-269, 1999.

[40] L. McIntosh, R. Summers, M. Jackson, H. Mantsch, J. Mansfield, M. Howlett, A. Crowson, and J. Toole, "Towards non-invasive screening of skin lesions by near-infrared spectroscopy," J. Invest. Dermatol., vol. 116, pp. 175-181, 2001.

[41] C. Frank, D. Redd, T. Gansler, and R. McCreery, "Characterization of human breast biopsy specimens with near-IR Raman spectroscopy," Anal. Chem., vol. 66, pp. 319-326, 1994.

[42] Z. Huang, A. McWilliams, H. Lui, D. McLean, S. Lam, and H. Zeng, "Near-infrared Raman spectroscopy for optical diagnosis of lung cancer," Int. J. Cancer, vol. 107, pp. 1047-1052, 2003.

[43] M. Chowdary, K. Kumar, J. Kurien, S. Mathew, and C. Krishna, "Discrimination of normal, benign, and malignant breast tissues by Raman spectroscopy," Biopolymers, vol. 83, pp. 556-569, 2006.

[44] A. Robichaux-Viehoever, E. Kanter, H. Shappell, D. Billheimer, H. Jones, III, and A. Mahadevan-Jansen, "Characterization of Raman spectra measured in vivo for the detection of cervical dysplasia," Appl. Spectrosc., vol. 61, pp. 986-993, 2007.

[45] T. Wang, G. Triadafilopoulos, J. Crawford, L. Dixon, T. Bhandari, P. Sahbaie, S. Friedland, R. Soetikno, and C. Contag, "Detection of endogenous biomolecules in Barrett's esophagus by Fourier transform infrared spectroscopy," Proc. Nat. Acad. Sci., vol. 104, pp. 158-64, 2007.

[46] A. Oliveira, R. Bitar, L. S. Jr, R. Zangaro, and A. Martin, "Near-infrared Raman spectroscopy for oral carcinoma diagnosis," Photomed. Laser Therapy, vol. 24, pp. 348-353, 2006.

[47] R. Levenson, "Spectral imaging perspective on cytomics," Cytometry, vol. 69A, pt. A, pp. 592-600, 2006.

[48] F. Woolfe, M. Maggioni, G. Davis, F. Warner, R. Coifman, and S. Zucker, Hyper-spectral microscopic discrimination between normal and cancerous colon biopsies [Online]. Available: http://www.math.duke.edu/ mauro/Papers/ColonCarcinoma (IEEE pdf)F. Woolfe, M. Maggioni, G. Davis, F. Warner, R. Coifman, and S. Zucker, "Hyper-spectral microscopic discrimination between normal and cancerous colon biopsies," IEEE Trans. Med. Imag.

[49] L. Boucheron, Z. Bi, N. Harvey, B. Manjunath, and D. Rimm, "Utility of multispectral imaging for nuclear classification of routine clinical histopathology imagery," BMC Cell Biol., vol. 8, p. S8, 2007.

[50] K. Masood and N. Rajpoot, "Classification of hyperspectral colon biopsy images: Does 2D spatial analysis suffice?" Ann. BMVA, vol. 2008, no. 4, pp. 1-15, 2008.

[51] A. Stadlbauer, S. Gruber, C. Nimsky, R. Fahlbusch, T. Hammen, R. Buslei, B. Tomandl, E. Moser, and O. Ganslandt, "Preoperative grading of gliomas by using metabolite quantification with high-spatial-resolution proton MR spectroscopic imaging," Radiology, vol. 238, pp. 958-969, Mar. 2006.

[52] P. Tiwari, A. Madabhushi, and M. Rosen, "A hierarchical spectral clustering and nonlinear dimensionality reduction scheme for detection of prostate cancer from magnetic resonance spectroscopy (MRS)," Med. Phys., vol. 36, no. 9, pp. 3927-3939, Sep. 2009.

[53] L. Yang, P. Meer, and D. Foran, "Unsupervised segmentation based on robust estimation and color active contour models," IEEE Trans. Inf. Technol. Biomed., vol. 9, no. 3, pp. 475-486, Sep. 2005.

[54] H. Narasimha-Iyer, A. Can, B. Roysam, V. Stewart, H. Tanenbaum, A. Majerovics, and H. Singh, "Robust detection and classification of longitudinal changes in color retinal fundus images for monitoring diabetic retinopathy," IEEE Trans. Biomed. Eng., vol. 53, no. 6, pp. 1084-1098, Jun. 2006
[55] M. Bello, A. Can, and X. Tao, "Accurate registration and failure detection in tissue micro array images," in 5th IEEE Int. Symp. Biomed. Imag.: From Nano to Macro, 2008, pp. 368-371.

[56] D. F. Gleason, "Histologic grading of prostate cancer: A perspective," Hum. Pathol., vol. 23, pp. 273-9, Mar. 1992.

[57] G. Alexe, G. S. Dalgin, D. Scanfeld, P. Tamayo, J. P. Mesirov, C. DeLisi, L. Harris, N. Barnard, M. Martel, A. J. Levine, S. Ganesan, and G. Bhanot, "High expression of lymphocyte-associated genes in node-negative HER2+ breast cancers correlates with lower recurrence rates," Cancer Res., vol. 67, pp. 10669-76, Nov. 2007.

[58] M. Bibbo, D. H. Kim, T. Pfeifer, H. E. Dytch, H. Galera-Davidson, and P. H. Bartels, "Histometric features for the grading of prostatic carcinoma," Anal. Quant. Cytol. Histol., vol. 13, pp. 61-8, Feb. 1991.

[59] J. A. Belien, J. P. Baak, P. J. van Diest, and A. H. van Ginkel, "Counting mitoses by image processing in Feulgen stained breast cancer sections: The influence of resolution," Cytometry, vol. 28, pp. 135-40, Jun. 1997.

[60] T. Markiewicz, S. Osowski, J. Patera, and W. Kozlowski, "Image processing for accurate cell recognition and count on histologic slides," Anal. Quant. Cytol. Histol., vol. 28, pp. 281-91, Oct. 2006.

[61] Y. J. Kim, B. F. Romeike, J. Uszkoreit, and W. Feiden, "Automated nuclear segmentation in the determination of the Ki-67 labeling index in meningiomas," Clin. Neuropathol., vol. 25, pp. 67-73, Mar.-Apr. 2006.

[62] J. K. Sont, W. I. De Boer, W. A. van Schadewijk, K. Grunberg, J. H. van Krieken, P. S. Hiemstra, and P. J. Sterk, "Fully automated assessment of inflammatory cell counts and cytokine expression in bronchial tissue," Amer. J. Respir. Crit. Care Med., vol. 167, pp. 1496-503, Jun. 2003.

[63] S. Naik, S. Doyle, A. Madabhushi, J. Tomaszeweski, and M. Feldman, "Automated gland segmentation and Gleason grading of prostate histology by integrating low-, high-level and domain specific information," presented at the Workshop on Microscopic Image Analysis With Applications in Biology, Piscataway, NJ, 2007.

[64] P. S. Karvelis, D. I. Fotiadis, I. Georgiou, and M. Syrrou, "A watershed based segmentation method for multispectral chromosome images classification," in Conf. Proc. IEEE Eng. Med. Biol. Soc., 2006, vol. 1, pp. 3009-12.

[65] S. Petushi, F. U. Garcia, M. M. Haber, C. Katsinis, and A. Tozeren, "Large-scale computations on histology images reveal grade-differentiating parameters for breast cancer," BMC Med. Imag., vol. 6, p. 14, 2006.

[66] D. C. Fernandez, R. Bhargava, S. M. Hewitt, and I. W. Levin, "Infrared spectroscopic imaging for histopathologic recognition," Nature Biotechnol., vol. 23, pp. 469-74, Apr. 2005.

[67] L. E. Boucheron, Z. Bi, N. R. Harvey, B. Manjunath, and D. L. Rimm, "Utility of multispectral imaging for nuclear classification of routine clinical histopathology imagery," BMC Cell Biol., vol. 8, Suppl. 1, p. S8, 2007.

[68] S. S. Singh, B. Qaqish, J. L. Johnson, O. H. Ford 3rd, J. F. Foley, S. J. Maygarden, and J. L. Mohler, "Sampling strategy for prostate tissue microarrays for Ki-67 and androgen receptor biomarkers," Anal. Quant. Cytol. Histol., vol. 26, pp. 194-200, Aug. 2004.

[69] D. L. Weaver, D. N. Krag, E. A. Manna, T. Ashikaga, S. P. Harlow, and K. D. Bauer, "Comparison of pathologist-detected and automated computer-assisted image analysis detected sentinel lymph node micrometastases in breast cancer," Mod. Pathol., vol. 16, pp. 1159-63, Nov. 2003.

[70] D. Glotsos, P. Spyridonos, P. Petalas, D. Cavouras, P. Ravazoula, P. A. Dadioti, I. Lekka, and G. Nikiforidis, "Computer-based malignancy grading of astrocytomas employing a support vector machine classifier, the WHO grading system and the regular hematoxylin-eosin diagnostic staining procedure," Anal. Quant. Cytol. Histol., vol. 26, pp. 77-83, Apr. 2004.

[71] C. Wahlby, I. M. Sintorn, F. Erlandsson, G. Borgefors, and E. Bengtsson, "Combining intensity, edge and shape information for 2D and 3D segmentation of cell nuclei in tissue sections," J Microsc., vol. 215, pp. 67-76, Jul. 2004.

[72] V. R. Korde, G. T. Bonnema, W. Xu, C. Krishnamurthy, J. RangerMoore, K. Saboda, L. D. Slayton, S. J. Salasche, J. A. Warneke, D. S. Alberts, and J. K. Barton, "Using optical coherence tomography to evaluate skin sun damage and precancer," Lasers Surg. Med., vol. 39, pp. 687-95, Oct. 2007.

[73] M. Gurcan, T. Pan, H. Shimada, and J. H. Saltz, "Image analysis for neuroblastoma classification: Hysteresis thresholding for cell segmentation," in APIII 2006, Vancouver, BC, Canada, 2006. 
[74] M. N. Gurcan, T. Pan, H. Shimada, and J. Saltz, "Image analysis for neuroblastoma classification: segmentation of cell nuclei," in Conf. Proc. IEEE Eng. Med. Biol. Soc., 2006, vol. 1, pp. 4844-7.

[75] P. Bamford and B. Lovell, "Method for accurate unsupervised cell nucleus segmentation," in Proc. 23rd Annu. Int. Conf. IEEE Engineering in Medicine and Biology Society, 2001, vol. 3, pp. 2704-2708.

[76] G. Begelman, M. Pechuk, E. Rivlin, and E. Sabo, "System for computer-aided multiresolution microscopic pathology diagnostics," in IEEE Int. Conf. Computer Vision Systems, 2006, p. 16.

[77] S. Naik, S. Doyle, S. Agner, A. Madabhushi, J. Tomaszeweski, and M. Feldman, "Automated gland and nuclei segmentation for grading of prostate and breast cancer histopatholog," in ISBI Special Workshop on Computational Histopathology (CHIP), Paris, France, 2008, pp. 284-287.

[78] J. A. Sethian, Level Set Methods: Evolving Interfaces in Geometry, Fluid Mechanics, Computer Vision and Materials Sciences, First ed. Cambridge, U.K.: Cambridge University Press, 1996.

[79] J. Kong, H. Shimada, K. Boyer, J. H. Saltz, and M. Gurcan, "Image analysis for automated assessment of grade of neuroblastic differentiation," in IEEE Int. Symp. Biomedical Imaging (ISBI), Washington, DC, Apr. 12-15, 2007.

[80] K. Rodenacker and E. Bengtsson, "A feature set for cytometry on digitized microscopic images," Anal. Cell. Pathol., vol. 25, pp. 1-36, 2003.

[81] A. J. Sims, M. K. Bennett, and A. Murray, "Image analysis can be used to detect spatial changes in the histopathology of pancreatic tumours," Phys. Med. Biol., vol. 48, pp. N183-N191, 2003.

[82] J. Gil, H. Wu, and B. Y. Wang, "Image analysis and morphometry in the diagnosis of breast cancer," Microsc. Res. Tech., vol. 59, pp. 109-118, 2002.

[83] L. E. Boucheron, "Object- and Spatial-Level Quantitative Analysis of Multispectral Histopathology Images for Detection and Characterization of Cancer," Ph.D. dissertation, Univ. of California Santa Barbara, Santa Barbara, CA, 2008.

[84] O. Sertel, J. Kong, U. Catalyurek, G. Lozanski, J. Saltz, and M. Gurcan, "Histopathological image analysis using model-based intermediate representations and color texture: Follicular lymphoma grading," $J$. Signal Process. Syst., to be published.

[85] M. Faloutsos, P. Faloutsos, and C. Faloutsos, "On power-law relationships of the internet topology," in , 1999, pp. 251-262.

[86] D. Watts and S. Strogatz, "Collective dynamics of 'small-world' networks," Nature, vol. 393, pp. 440-442, 1998.

[87] S. Wuchty, E. Ravasz, and A. Barabasi, "The architecture of biological networks," in Complex Systems in Biomedicine, T. S. Deisboeck and J. Yasha Kresh, Eds. New York: Springer, 2003, pp. 165-176.

[88] R. Albert, T. Schindewolf, I. Baumann, and H. Harms, "Three-dimensional image processing for morphometric analysis of epithelium sections," Cytometry, vol. 13, pp. 759-765, 1992.

[89] C. Bilgin, C. Demir, C. Nagi, and B. Yener, "Cell-graph mining for breast tissue modeling and classification," in 29th Annu. Int. Conf. IEEE EMBS, Lyon, France, Aug. 22-26, 2007, pp. 5311-5314.

[90] C. Bilgin, P. Bullough, G. Plopper, and B. Yener, "ECM aware cellgraph mining for bone tissue modeling and analysis," RPI Computer Science, 2008, Tech. Rep. 08-07.

[91] C. Gunduz, B. Yener, and S. Gultekin, "The cell graphs of cancer," Bioinformatics, vol. 20, 2004.

[92] S. Doyle, M. Hwang, K. Shah, A. Madabhushi, M. Feldman, and J. Tomaszeweski, "Automated grading of prostate cancer using architectural and textural image features," in ISBI, 2007, pp. $1284-1287$.

[93] J. Kong, O. Sertel, H. Shimada, K. Boyer, J. Saltz, and M. Gurcan, "Computer-aided evaluation of neuroblatoma on whole-slide histology images: Classifying grade of neuroblastic differentiation," Pattern Recognit., vol. 42, pp. 1080-1092, 2009.

[94] O. Sertel, J. Kong, H. Shimada, U. Catalyurek, J. Saltz, and M. N. Gurcan, "Computer-aided prognosis of neuroblastoma on whole-slide images: Classification of stromal development," Pattern Recognit., vol. 42, pp. 1093-1103, 2009.

[95] P. Burt and E. Adelson, "The Laplacian pyramid as a compact image code," IEEE Trans. Commun., vol. 31, no. 4, pp. 532-540, Apr. 1983.

[96] S. Doyle, A. Madabhushi, M. Feldman, and J. Tomaszeweski, "A boosting cascade for automated detection of prostate cancer from digitized histology," Lecture Notes in Computer Science, vol. 4191, p. 504, 2006

[97] P. Pudil, J. Novovivcova, and J. Kittler, "Floating search methods in feature selection," Pattern Recognit. Lett., vol. 15, pp. 1119-1125, 1994.
[98] A. Jain and D. Zongker, "Feature selection: Evaluation, application, and small sample performance," IEEE Trans. Pattern Anal. Mach. Intell., vol. 19, no. 2, pp. 153-158, Feb. 1997.

[99] Y. Freund and R. E. Shapire, "A decision-theoretic generalization of on-line learning and an application to boosting," J. Comput. Syst. Sci., vol. 55, pp. 119-139, 1997.

[100] S. Perkins, K. Lacker, and J. Theiler, "Grafting: Fast, incremental feature selection by gradient descent in function space," J. Mach. Learn. Res., vol. 3, pp. 1333-1356, 2003.

[101] H. Qureshi, O. Sertel, N. Rajpoot, R. Wilson, and M. Gurcan, "Adaptive discriminant wavelet packet transform and local binary patterns for meningioma subtype classification," in Medical Image Computing and Computer-Assisted Intervention - MICCAI 2008, pp. 196-204.

[102] I. Jolliffe, Principal Component Analysis. New York: SpringerVerlag, 2002.

[103] A. Martinez and A. Kak, "PCA versus LDA," IEEE Trans. Pattern Anal. Mach. Intell., vol. 23, no. 2, pp. 228-233, Feb. 2001.

[104] A. Hyvärinen and E. Oja, "Independent component analysis: Algorithms and applications," Neural Netw., vol. 13, pp. 411-430, 2000.

[105] N. Rajpoot, M. Arif, and A. Bhalerao, "Unsupervised learning of shape manifolds," in Proc. British Machine Vision Conf., 2007, pp. 312-321.

[106] R. Coifman, S. Lafon, A. Lee, M. Maggioni, B. Nadler, F. Warner, and S. Zucker, "Geometric diffusions as a tool for harmonic analysis and structure definition of data: Diffusion maps," Proc. Nat. Acad. Sci., vol. 102, pp. 7426-7431, 2005

[107] L. I. Kuncheva and C. J. Whitaker, "Measures of diversity in classifier ensembles and their relationship with the ensemble accuracy," Mach. Learn., vol. 51, pp. 181-207, 2003.

[108] S. Doyle, Hwang, M. Shah, K. Madabhushi, A. Tomaszeweski, and J. M. Feldman, "Automated grading of prostate cancer using architectural and textural image features," in Int. Symp. Biomedical Imaging, Washington, DC, 2007, pp. 1284-87.

[109] K. Rajpoot and N. Rajpoot, "SVM optimization for hyperspectral colon tissue cell classification," in Medical Image Computing and ComputerAssisted Intervention-MICCAI 2004, pp. 829-837.

[110] S. Doyle, C. Rodriguez, A. Madabhushi, J. Tomaszeweski, and M. Feldman, "Detecting prostatic adenocarcinoma from digitized histology using a multi-scale, hierarchical classification approach," in IEEE Engineering in Medicine and Biology Conf., New York, NY, 2006, pp. 4759-62.

[111] P. Viola and M. J. Jones, "Robust real-time face detection," Int. J. Comput. Vis., vol. 57, pp. 137-154, May 2004

[112] C. Demir and B. Yener, "Automated cancer diagnosis based on histopathological images: A systematic survey,” Rensselaer Polytechnic Institute, Troy, NY, 2006.

[113] C. Gunduz, B. Yener, and S. H. Gultekin, "The cell graphs of cancer," Bioinformatics, vol. 20, Suppl. 1, pp. i145-51, Aug. 2004.

[114] K. Jafari-Khouzani and H. Soltanian-Zadeh, "Multiwavelet grading of pathological images of prostate," IEEE Trans. Biomed. Eng., vol. 50 , no. 6, pp. 697-704, Jun. 2003.

[115] B. Weyn et al., "Automated breast tumor diagnosis and grading based on wavelet chromatin texture description," Cytometry, vol. 33, pp. $32-40,1998$

[116] G. Van deWouwer, B. Weyn, P. Scheunders, W. Jacob, E. Van Marck, and D. VAN, "Wavelets as chromatin texture descriptors for the automated identification of neoplastic nuclei," J. Microscopy, vol. 197, p. $25,2000$.

[117] A. Tabesh, M. Teverovskiy, H. Y. Pang, V. P. Kumar, D. Verbel, A. Kotsianti, and O. Saidi, "Multifeature prostate cancer diagnosis and Gleason grading of histological images," IEEE Trans. Med. Imag., vol. 26, no. 10 , pp. 1366-1378, Oct. 2007

[118] A. N. Esgiar, R. N. Naguib, B. S. Sharif, M. K. Bennett, and A. Murray, "Microscopic image analysis for quantitative measurement and feature identification of normal and cancerous colonic mucosa," IEEE Trans. Inf. Technol. Biomed., vol. 2, no. 3, pp. 197-203, Sep. 1998.

[119] S. Keenan, J. Diamond, W. G. McCluggage, H. Bharucha, D. Thompson, P. Bartels, and P. Hamilton, "An automated machine vision system for the histological grading of cervical intraepithelial neoplasia (CIN)," J Pathol., vol. 192, no. 3, pp. 351-362, 2000.

[120] B. Cambazoglu, O. Sertel, J. Kong, J. H. Saltz, M. N. Gurcan, and U. V. Catalyurek, "Efficient processing of pathological images using the grid: Computer-aided prognosis of neuroblastoma," in Proc. Fifth Int. Workshop on Challenges of Large Applications in Distributed Environments, Monterey Bay, CA, 2007, pp. 35-41.

[121] A. Ruiz, O. Sertel, M. Ujaldon, U. V. Catalyurek, J. Saltz, and M. N. Gurcan, "Pathological image analysis using the GPU: Stroma classification for neuroblastoma," in IEEE BIBM'07, 2007. 
[122] M. N. Gurcan, H. P. Chan, B. Sahiner, L. Hadjiiski, N. Petrick, and M. A. Helvie, "Optimal neural network architecture selection: Improvement in computerized detection of microcalcifications," Acad. Radiol., vol. 9, pp. 420-9, Apr. 2002.

[123] J. Chappelow, S. Viswanath, A. Madabhushi, M. Rosen, J. Tomaszeweski, and M. Feldman, "Improving supervised classification accuracy using non-rigid multimodal image registration: Computer-aided detection of prostate cancer on ex vivo MRI," in SPIE Medical Imaging, San Diego, CA, 2008.

[124] J. Chappelow, A. Madabhushi, M. Rosen, J. Tomaszeweski, and M. Feldman, "Multimodal image registration of ex vivo 4 tesla prostate MRI with whole mount histology for cancer detection," in SPIE Medical Imaging, San Diego, 2007, vol. 6512(1), pp. S1-S12.

[125] S. Ourselin, A. Roche, G. Subsol, X. Pennec, and N. Ayache, "Reconstructing a 3D structure from serial histological sections," Image Vis. Comput., vol. 19, pp. 25-31, 2001.

[126] A. Madabhushi, M. Feldman, D. Metaxas, J. Tomaszeweski, and D. Chute, "Automated detection of prostatic adenocarcinoma from high resolution ex vivo MRI," IEEE Trans. Med. Imag., vol. 24, no. 12, pp. 1611-1625, Dec. 2005.

[127] M. Teverovskiy, Y. Vengrenyuk, A. Tabesh, M. Sapir, S. Fogarasi, P. Ho-Yuen, F. M. Khan, S. Hamann, P. Capodieci, M. Clayton, R. Kim, G. Fernandez, R. Mesa-Tejada, and M. J. Donovan, "Automated localization and quantification of protein multiplexes via multispectral fluorescence imaging," in 5th IEEE Int. Symp. Biomedical Imaging: From Nano to Macro, 2008, pp. 300-303.

[128] E. Glory and R. F. Murphy, "Automated subcellular location determination and high-throughput microscopy," Develop. Cell, vol. 12, pp. $7-16,2007$.

[129] B. Lessmann, A. Degenhard, P. Kessar, L. Pointon, M. Khazen, M. Leach, and T. Nattkemper, "SOM-based wavelet filtering for the exploration of medical images," in Artificial Neural Networks: Biological Inspirations - ICANN 2005, 2005, pp. 671-676.

[130] J. R. Iglesias-Rozas and N. Hopf, "Histological heterogeneity of human glioblastomas investigated with an unsupervised neural network (SOM)," Histol. Histopathol., vol. 20, pp. 351-6, Apr. 2005.

[131] S. Doyle, M. Hwang, S. Naik, M. Feldman, J. Tomaszeweski, and A. Madabhushi, "Using manifold learning for content-based image retrieval of prostate histopathology," in MICCAI, 2007.

[132] A. Basavanhally, S. Agner, G. Alexe, G. Bhanot, S. Ganesan, and A. Madabhushi, "Manifold learning with graph-based features for identifying extent of lymphocytic infiltration from high grade breast cancer histology," presented at the MMBIA Workshop, 2008.

[133] G. Lexe, J. Monaco, S. Doyle, A. Basavanhally, A. Reddy, M. Seiler, S. Ganesan, G. Bhanot, and A. Madabhushi, "Towards improved cancer diagnosis and prognosis using analysis of gene expression data and computer aided imaging," Exp. Biol. Med., vol. 234, pp. 860-879, Aug. 2009.

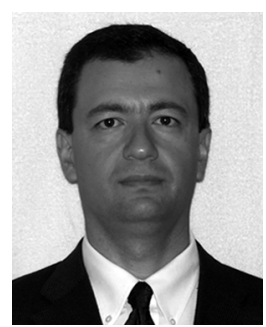

Metin N. Gurcan (S'90-M'99-SM'06) received the BSc. and Ph.D. degrees in electrical and electronics engineering from Bilkent University, Ankara, Turkey and the MSc. degree in digital systems engineering from the University of Manchester Institute of Science and Technology, Manchester, U.K.

During the winters of 1996 and 1997 he was a visiting Researcher at the University of Minnesota, Minneapolis. From 1999 to 2001, he was a Postdoctoral Research Fellow and later a Research Investigator in the Department of Radiology at the University of Michigan, Ann Arbor. He is now an Assistant Professor in the Biomedical Informatics Department of The Ohio State University, Columbus. Prior to joining the Ohio State University in October 2005, he worked as a Senior Researcher and Product Director at a high-tech company, specializing in computer-aided detection and diagnosis of cancer from radiological images. He currently manages several image analysis related projects funded by the National Cancer Institute, National Library of Medicine, American Cancer Society, Department of Defense, and Children's Neuroblastoma Cancer Foundation. He teaches tutorials at IEEE, ICIP, and ICASSP conferences, and is the organizer of and lecturer at several meetings, workshops, and special focus sessions at leading conferences in areas of image analysis.

Dr. Gurcan is a senior member of SPIE and RSNA. He is the recipient of the British Foreign and Commonwealth Organization Award, Children's Neuroblastoma Cancer Foundation Young Investigator Award, and National Cancer Institute's caBIG Embodying the Vision Award.

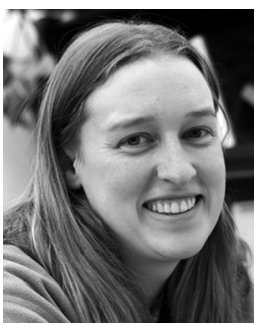

Laura E. Boucheron (S'01-M'07) received the B.S. degree in electrical engineering and the M.S. degree in electrical engineering from New Mexico State University, Las Cruces, NM, in 2001 and 2003, respectively, and the $\mathrm{Ph} . \mathrm{D}$. degree in electrical and computer engineering from the University of Santa Barbara, Santa Barbara, CA, in 2008.

She is currently a Postdoctoral Fellow at New Mexico State University in the Klipsch School of Electrical and Computer Engineering. She has previous intern and graduate research assistant experience at both Sandia National Laboratories and Los Alamos National Laboratory. Her main research interests include high-level image analysis and pattern classification, with special interest in pathology imagery (histo- and cyto-pathology).

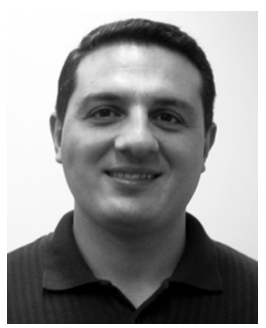

Ali Can received the B.S. degree in electrical engineering from the University of Gaziantep, Turkey, in 1993, and the M.S. degree in electrical engineering and the Ph.D. degree in computer and systems engineering from Rensselaer Polytechnic Institute, Troy, NY, in 1997 and 2000, respectively.

He has been a Researcher at the Visualization and Computer Vision Laboratory, General Electric (GE) Global Research Center, Niskayuna, NY, since 2004. Before joining GE, he was a Postdoctoral Researcher at Woods Hole Oceanographic Institution, Woods Hole, MA. He has been working on diverse machine vision application areas from industrial inspection to medical imaging, and from life science applications to personalized medicine. His research interests include image enhancement, analysis, registration, and segmentation, quantitation, limited angle tomography, inverse problems, and statistics. Some of the application areas of his work include molecular cell biology for cancer research, molecular pathology, histopathology, quantitation of Parkinson's disease, mammography, nondestructive testing of industrial parts, underwater imaging, 3-D confocal microscopy, and retinal fundus imaging. In Summer 2003, he participated in an underwater archaeology cruise in the Black Sea with Dr. R. Ballard, and a deepwater coral reefs research in the U.S. Virgin Islands with Dr. H. Singh.

Dr. Can received the Best Paper Award at the 1999 IEEE Conference on Computer Vision and Pattern Recognition. He received the Microscopy Society of America Presidential Student Award, and the Allen B. Dumont Prize for his doctoral work, both in 2000 .

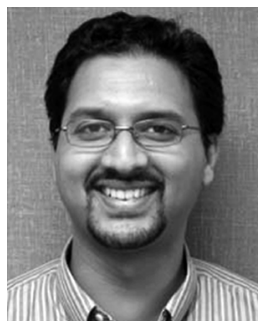

Anant Madabhushi (S'98-M'04-SM'09) received the B.S. degree in biomedical engineering from Mumbai University, India, in 1998, the M.S. degree in biomedical engineering from the University of Texas, Austin, in 2000, and the Ph.D. degree in bioengineering from the University of Pennsylvania in 2004.

He is the Director of the Laboratory for Computational Imaging and Bioinformatics (LCIB) in the Department of Biomedical Engineering, Rutgers University, Piscataway, NJ. He joined the Department of Biomedical Engineering, Rutgers University, as an Assistant Professor in 2005. $\mathrm{He}$ is also a member of the Cancer Institute of New Jersey and an Adjunct Assistant Professor of Radiology at the Robert Wood Johnson Medical Center, NJ. He has over 60 publications and book chapters in leading international journals and peer-reviewed conferences and patents pending in the areas of medical image analysis, computer-aided diagnosis, machine learning, and computer vision and in applying these techniques for early detection and diagnosis of prostate and breast cancer from high-resolution MRI, MR spectroscopy, protein- and gene-expression studies, and digitized tissue histopathology.

Dr. Madabhushi is the recipient of a number of awards for both research as well as teaching, including the Busch Biomedical Award (2006), the Technology Commercialization Award (2006), the Coulter Phase 1 and Phase 2 Early Career award (2006, 2008), the Excellence in Teaching Award (2007-2009), the Cancer Institute of New Jersey New Investigator Award (2007), the Society for Imaging Informatics in Medicine (SIIM) New Investigator award (2008), and the Life Sciences Commercialization Award (2008). In addition his research work has also received grant funding from the National Cancer Institute (NIH), New Jersey Commission on Cancer Research, and the Department of Defense. 


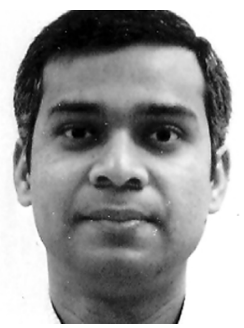

Nasir M. Rajpoot (S'98-M'01) was born in Multan, Pakistan. He received the B.S. degree in mathematics and the M.S. degree in computer science from Bahauddin Zakariya University, Pakistan, in 1991 and 1994, respectively, the M.S. degree in systems engineering from Quaid-e-Azam University, Pakistan, in 1996, and the Ph.D. degree in computer science from the University of Warwick, Coventry, U.K., in 2001. During 1998-2000 he was a visiting student at the Applied Mathematics program of Yale University, New Haven, CT.

He joined the University of Warwick as a Lecturer in computer science in 2001. Since 2006, he has been an Associate Professor in computer science at the University of Warwick. He has published on image coding and denoising. His current research interests are histology image analysis, texture analysis, multiresolution representations, and pattern analysis.

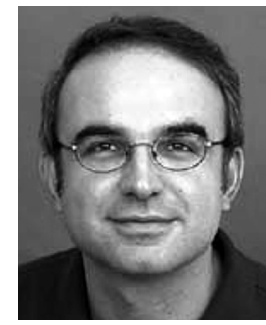

Bülent Yener (S'94-M'97-SM'03) received the MS. and Ph.D. degrees in computer science from Columbia University, New York, NY, in 1987 and 1994, respectively.

$\mathrm{He}$ is a Professor in the Department of Computer Science and Co-Director of Pervasive Computing and Networking Center at Rensselaer Polytechnic Institute (RPI), Troy, NY. Before joining RPI, he was a Member of Technical Staff at Bell Laboratories, Murray Hill, NJ. His current research interests include bioinformatics, medical informatics, and security and privacy. He has served on the Technical Program Committees of leading IEEE conferences and workshops.

Prof. Yener is an associate editor of ACM/Kluwer Wireless Networks Journal (Winet) and the IEEE Network Magazine. He is a member of the IEEE Communications and Computer Society. 